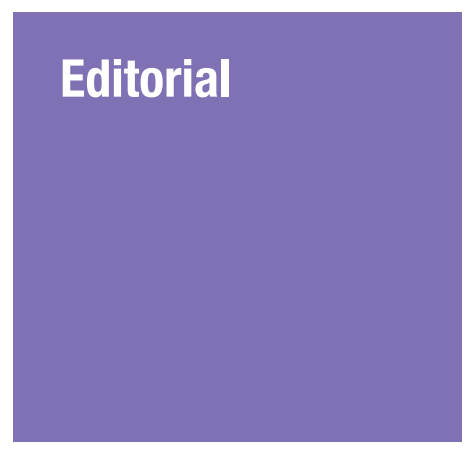

\title{
Neurosciences at Universiti Sains Malaysia Represent Malaysia to Support Global Precision Brain Health
}

\author{
Jafri Malin AbDuLLAH \\ Malaysian Journal of Medical Sciences, Universiti Sains Malaysia Health \\ Campus, Kubang Kerian, Kelantan, Malaysia
}

Submitted: 10 May 2019 Accepted: 9 Jun 2019 Online: 28 Jun 2019

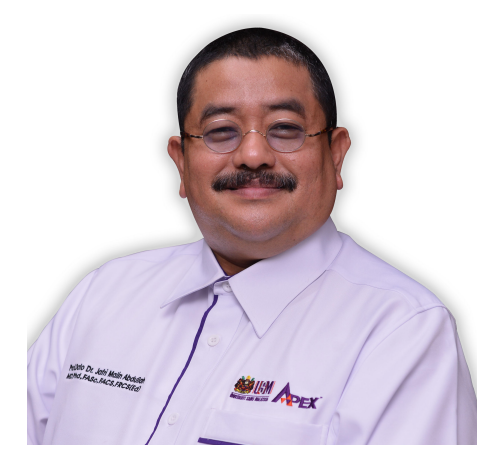

To cite this article: Abdullah JM. Neurosciences at Universiti Sains Malaysia represent Malaysia to support global precision brain health. Malays J Med Sci. 2019;26(3):1-23. https://doi.org/10.21315/mjms2019.26.3.1

To link to this article: https://doi.org/10.21315/mjms2019.26.3.1

\begin{abstract}
The combined effort of the neuroscience and psychology cluster at the Universiti Sains Malaysia (USM)-fundamental, applied and clinical-has moved the institution to the number two position in the country, behind Universiti Malaya. The strategy to join the Global Brain Consortium (GBC) and put Malaysia on the map to address the GBC mission, vision, focus areas and outcomes began recently, in May 2019.
\end{abstract}

Keywords: global, precision, brain, health, Malaysia, neurosciences, neurosurgery, psychology

\section{Introduction}

Malaysia was honoured to be invited to attend the World Health Organization (WHO)-supported Global Brain Consortium (GBC) workshop with the motto 'Towards Global Precision Brain Health' at the Montreal Neurological Institute's de Grandpré Communications Centre in Montreal, Canada from 9-10 May 2019. The topics discussed ranged from the future expectations of the WHO in terms of global brain health, international and multilateral research-funding agencies, datasharing infrastructures, and data governance for Electroencephalography (EEG) to studying dynamic brain starts with EEG, clinical research opportunities for EEG, clinical care translation using EEG, and the role of funders in advancing global brain collaborations.

\section{USM Moves to the International Level}

The GBC is a diverse network of brain researchers, clinicians and institutions committed to achieving improved and more equitable health outcomes worldwide. It is a Canada-based initiative that builds collaborative and dynamic relationships among globaloriented health researchers, domestically and internationally. 
The GBC will address the challenges facing multinational collaborative efforts in support of Global Precision Brain Health. It aims to strengthen linkages between like-minded neuroscientists across borders and disciplines to build a fluid and connected global research community that can advance equitable solutions to priority health challenges worldwide, with an orientation towards brain health and research challenges in low- and middle-income countries (LMICs) and guided by the overarching goal of reducing disparities in health outcomes (1).

Malaysia has prepared itself by establishing training programmes via Universiti Sains Malaysia's graduate-level courses that consider the United Nations' Sustainable Development Goals, especially in education. Global brain health requires trained medical and non-medical personnel combining both the arts and the sciences (2-6).

The Master of Surgery (Neurosurgery) programme of the Universiti Sains Malaysia is training future neurosurgeons (Figure 1) and has thus far graduated 78 neurosurgeons (Figure 2) since this 2-plus-4-year postgraduate coursework was initiated in 2001, currently coordinated by Professor Zamzuri Idris, Professor Dato' Dr Jafri Malin Abdullah, Associate Professor Abdul Rahman Izaini Ghani and Dr Regunath Kandasamy. The established Integrated Neuroscience Programme (INP), coordinated by $\mathrm{Dr}$ Muzaimi Mustapha, is on its eleventh batch of postgraduate students, producing both Masters and Doctors of Neurosciences since its establishment five-and-a-half years ago (Figure 3). The Master of Neurosciences by pure research, master coursework in neurology, and Doctorate in Neurosciences currently coordinated by Dr Farizan Ahmad has produced more than 35 graduates (Figure 4).

The newly established Integrated Clinical Psychology USM-UPSI courses coordinated by Associate Professor Dr Azizah Othman and Dr Mohamed Faiz Mohamed Mustafar and the Master of Cognitive Neuroscience courses coordinated by Professor Dato' Dr Jafri Malin Abdullah and Dr Aini Ismafairus Abd Hamid received their first batches of students in September 2018 and the second batch of Cognitive Neuroscience students in February 2019 (Figures 5 and 6). The newly established Brain Behaviour Cluster (Figure 7) has jumpstarted its activities, focusing on translational, transdisciplinary excellence in clinical neurology, neurosurgery, and psychology, with at least 56 members in various fields. Thus, we hope the ranking of clinical neurosciences and psychology will improve over the next three years in Malaysia, as per Figures 8 and 9. The Global Brain Consortium that Malaysia was invited to join will further focus on collaboration with other under developed and near developed countries to improve their neurological and mental healthcare using neurotechnology like the EEG (Figure 10).

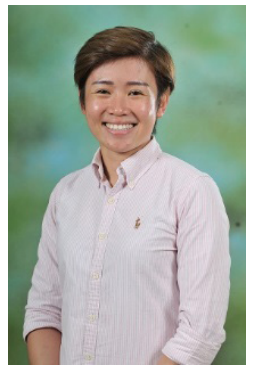

1)

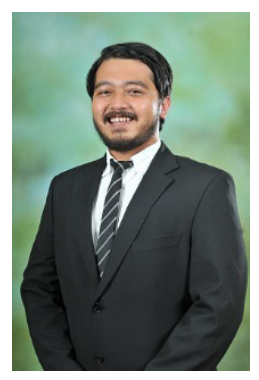

7)

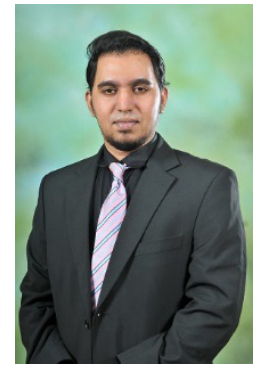

2)

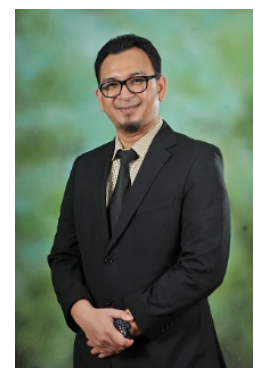

8)

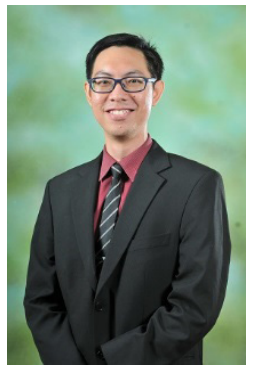

3)

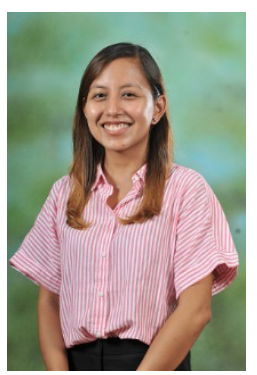

9)

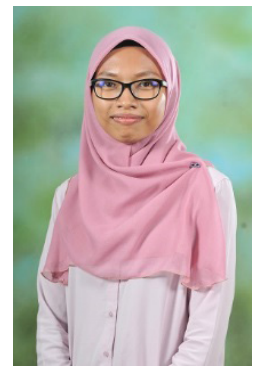

4)

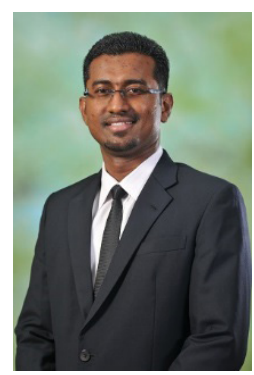

10)

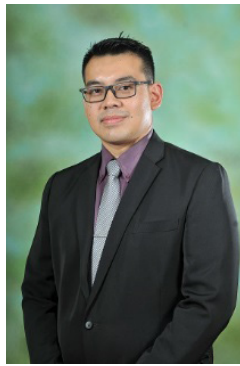

5)

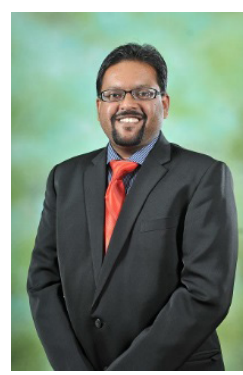

11)

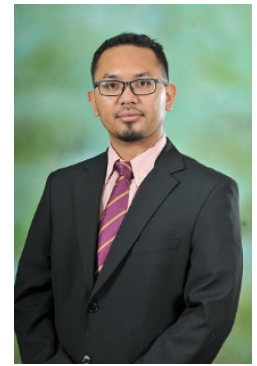

6)

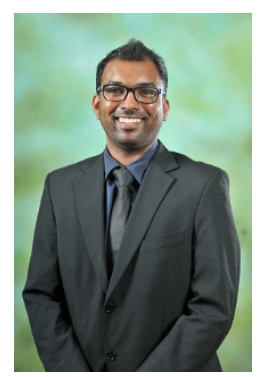

12) 
Editorial | Neurosciences Malaysia global precision brain health

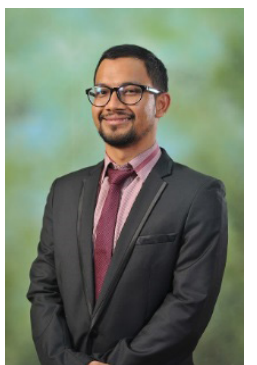

13)

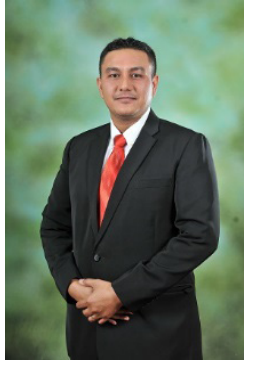

19)

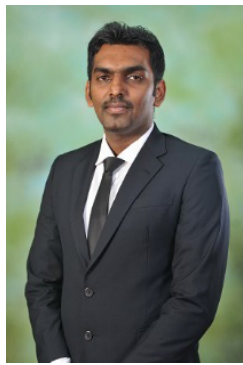

25)

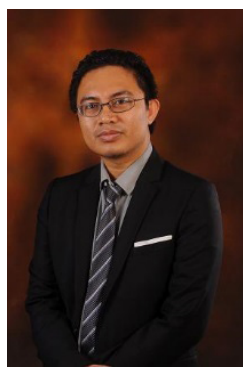

31)

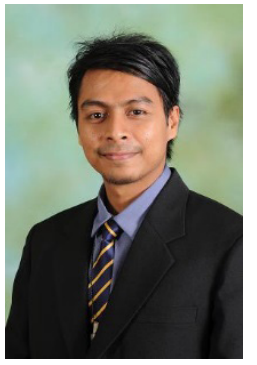

37)

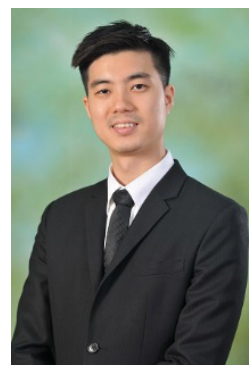

14)

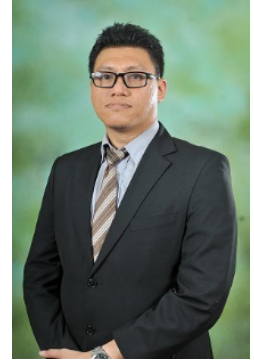

20)

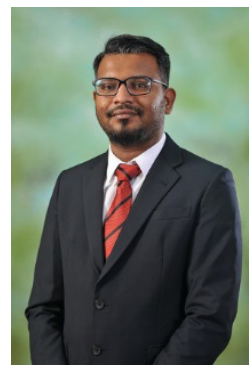

26)

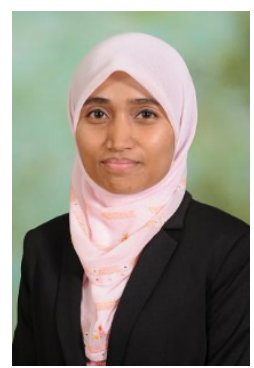

32)

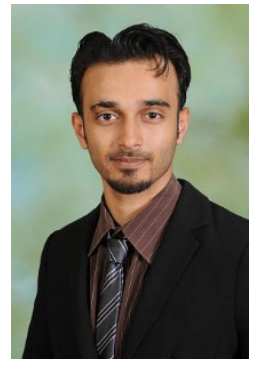

38)

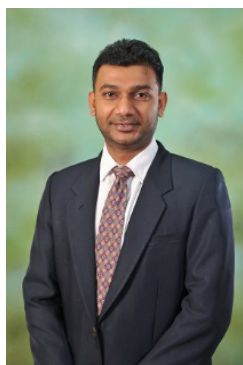

15)

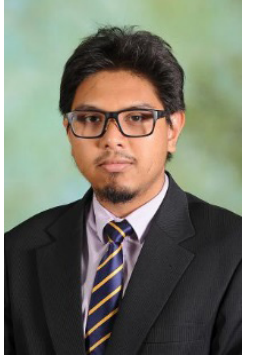

21)

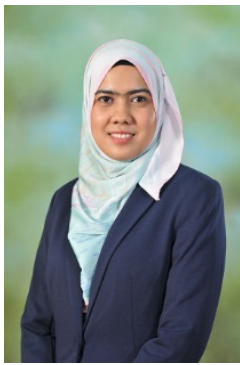

27)

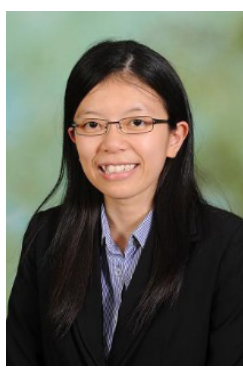

33)

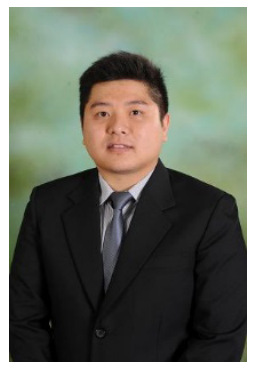

39)

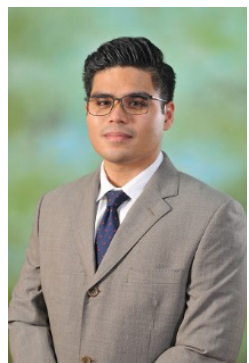

16)

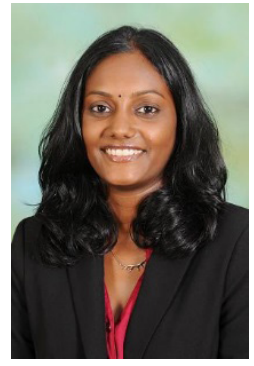

22)

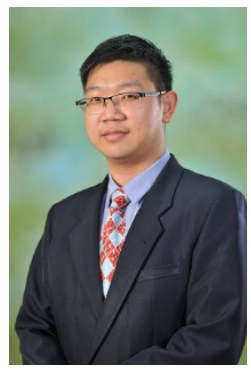

28)

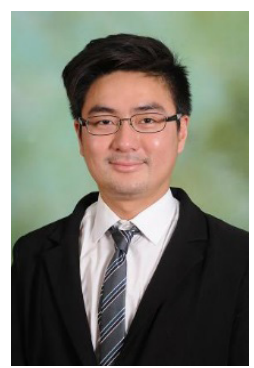

34)

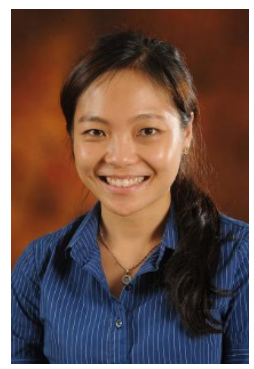

40)

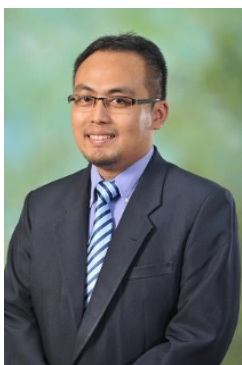

17)

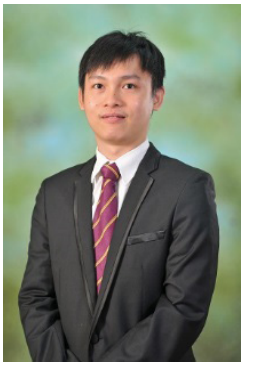

23)

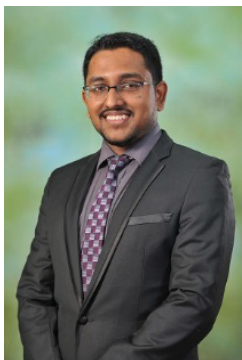

29)

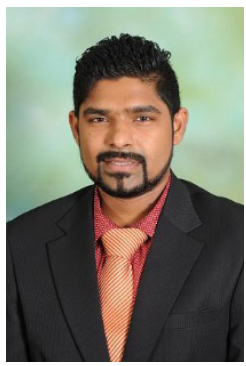

35)

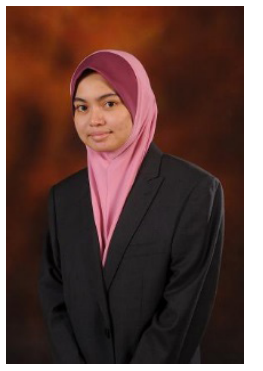

41)

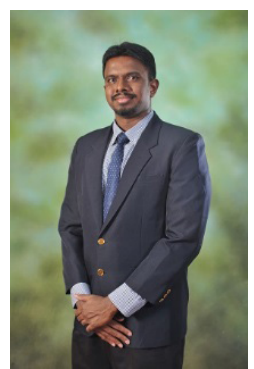

18)

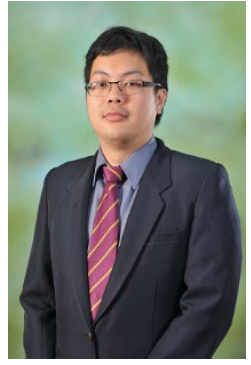

24)

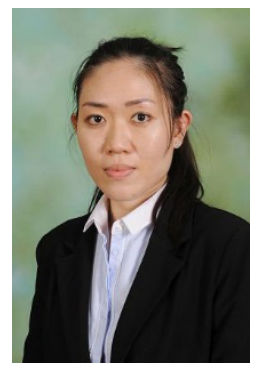

30)

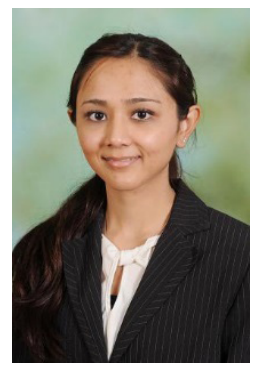

36)

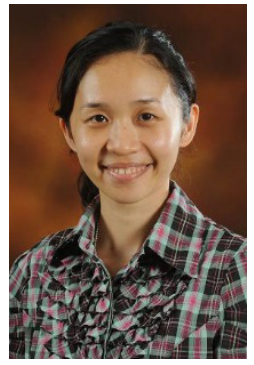

42) 


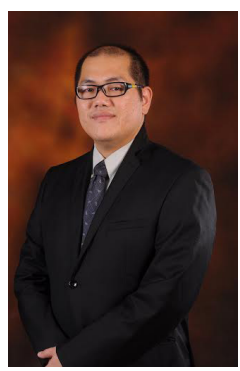

43)

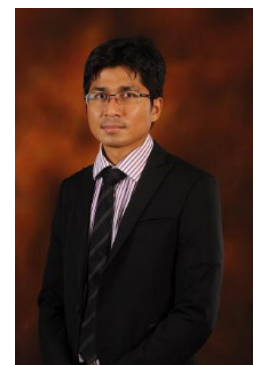

44)

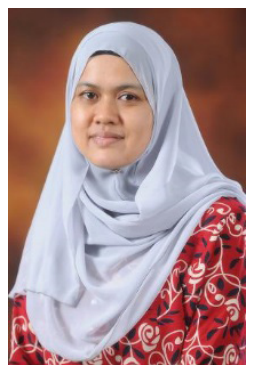

45)

Figure 1. Master of Surgery (Neurosurgery) residents till May 2019

Year 1

1) Dr Debbie Kong Ching Ching

2) Dr Harvinth Nagalingam Muniandy

3) Dr Looi Mun Choon

4) Dr Mas Syazaneeza Shab

5) Dr Mohd Farhan Mohd Faiz Wilson Yeo

6) Dr Mohd Ghadafi Wahab

7) Dr Mohd Iryan Che Othman

8) Dr Muhamad Ridzuan Alias

9) Dr Nadiah Ahmad Fuad

10) Dr Nishan Rao Subramaniam

11) Dr Rohan Jeevaraj

12) Dr V Jeyaseelan G Vasanthakumaran

13) Dr Vicnesh Thillynathan

14) Dr Zaharul Azran Zahari

Year 2

15) Dr Is Shahrom

16) Dr Julian Tan Li Kwang

17) Dr Kuha Raj a/l Arumugam

18) Dr Mohd Hezry Abu Hassan

19) Dr Mohd Khairun Mohd Mispan

20) Dr Thavanesan a/l Puvanesavaran Year 3

21) Dr Ahmad Zulfadli Mohamed Radzi

22) Dr Alarmelu Nithya Ramanathan
23) Dr Ang Song Yee

24) Dr Jesse Ze Ngai

25) Dr Kumarappan a/l Chokalingam

26) Dr Moventhiran a/l Ramakrishnan

27) Dr Nurshaheda Mohd Salleh

28) Dr Sam Joe Ee

29) Dr Saravanan a/l Sridharan

30) Dr Lim Mei Sin

31) Dr Mohd Najmi Abd Halim

32) Dr Sarah 'Atiqah Mohd Zamri

33) Dr Tan Shze Ee

34) Dr Teo Eu Gene

Year 4

35) Dr Arulkanesh Devatathan

36) Dr Diana Noma Fitzrol

37) Dr Muhammad Aizzat Othman

38) Dr Razmeender Singh Kelly

39) Dr Tan Zi Han

40) Dr Lee Chun Lin

41) Dr Shukriyah Sulong

42) Dr Lee Shwu Yi

43) Dr Lau Bik Liang

44) Dr Asrarul Fikri Abu Hassan

45) Dr Asmaa Mohamad Afifi 
Editorial | Neurosciences Malaysia global precision brain health

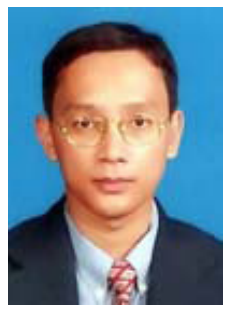

1)

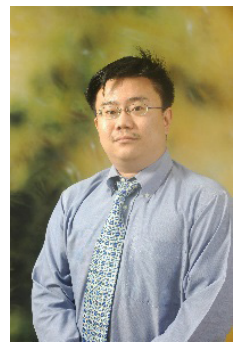

7)

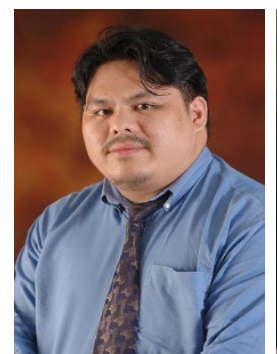

13)

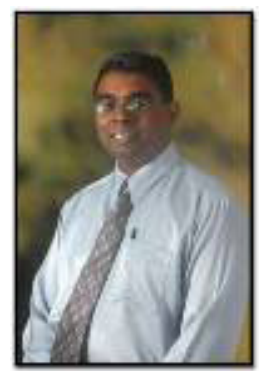

19)

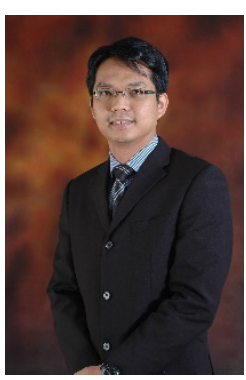

25)

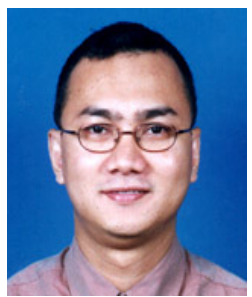

2)

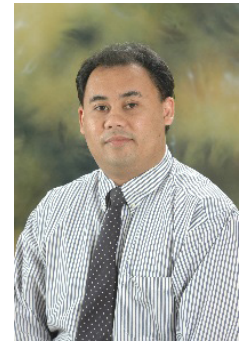

8)

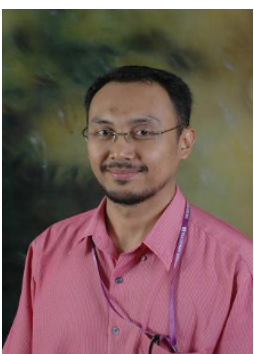

14)

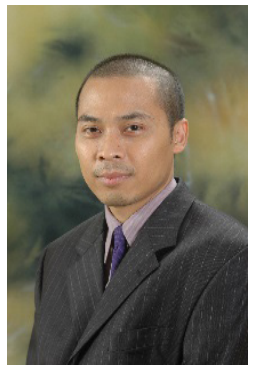

20)

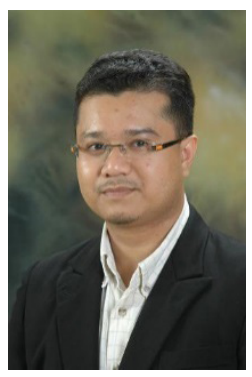

26)

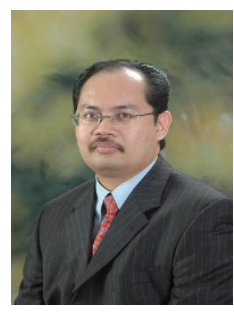

3)

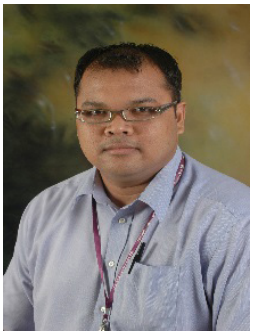

9)

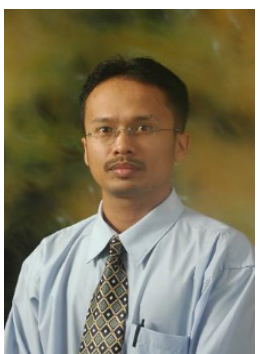

15)

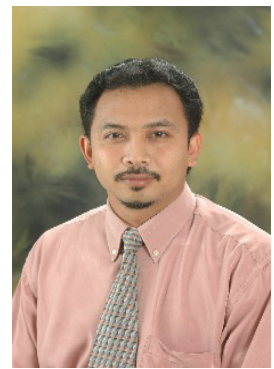

21)

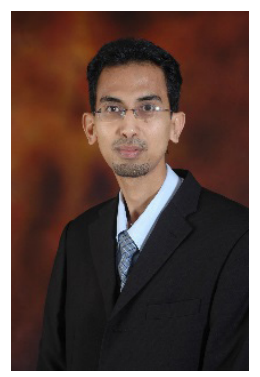

27)

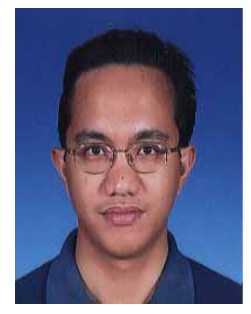

4)

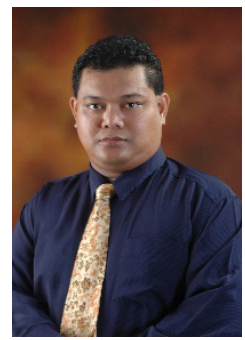

10)

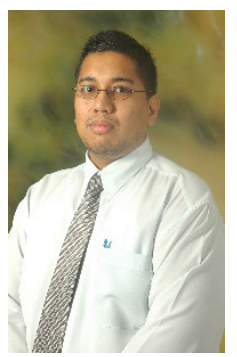

16)

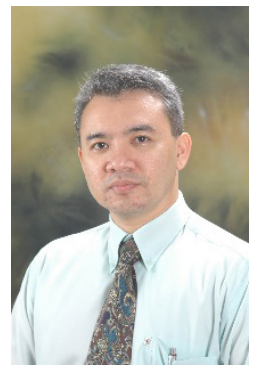

22)

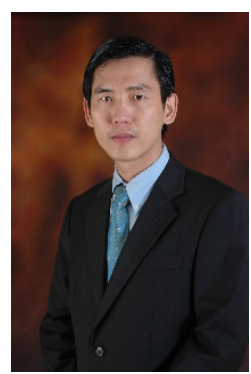

28)

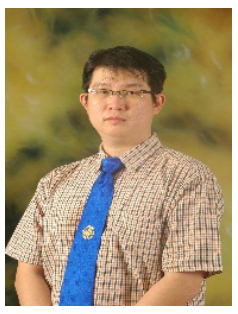

5)

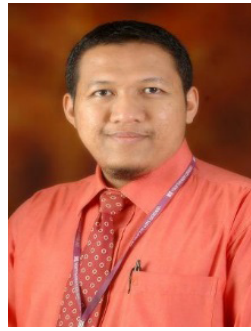

11)

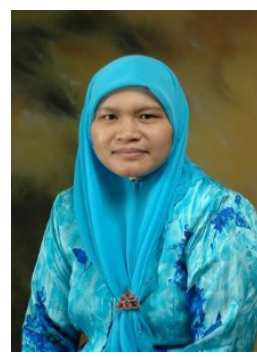

17)

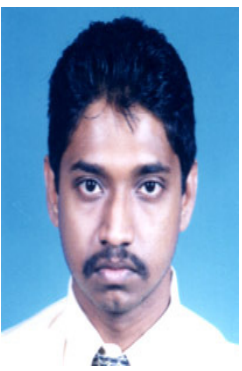

23)

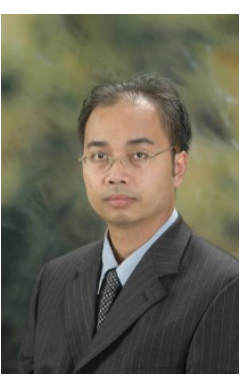

29)

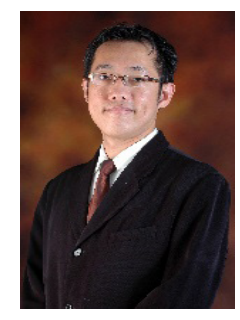

6)

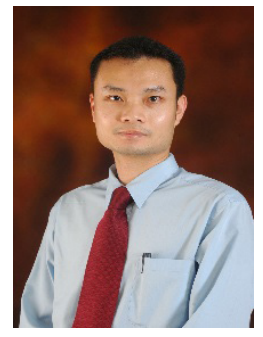

12)

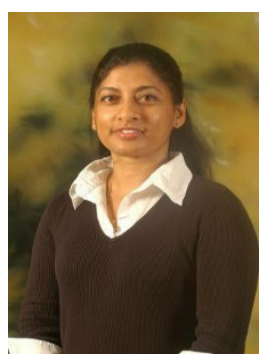

18)

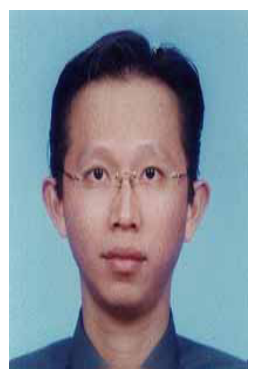

24)

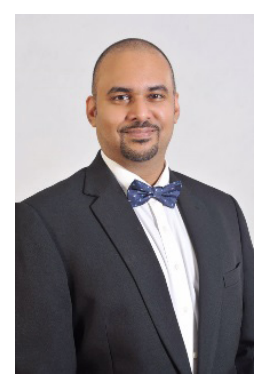

30) 


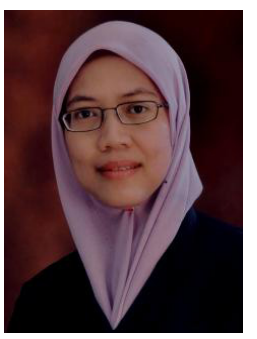

31)

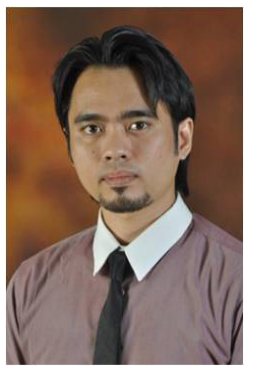

37)

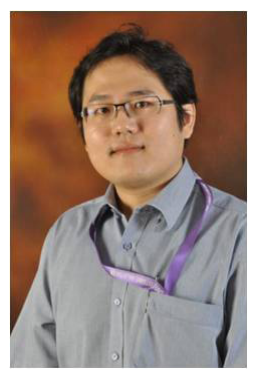

43)

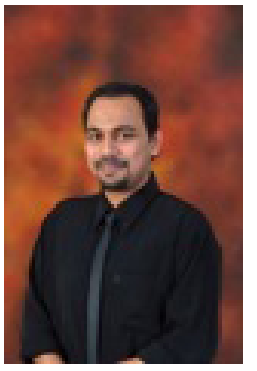

49)

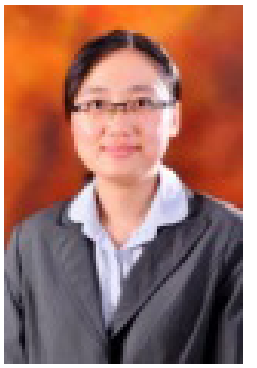

55)

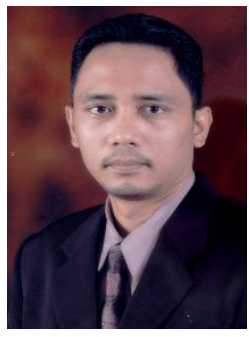

32)

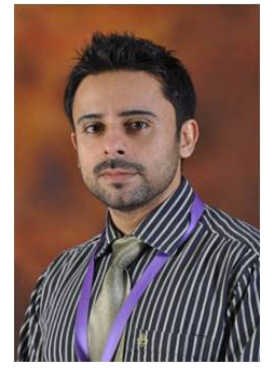

38)

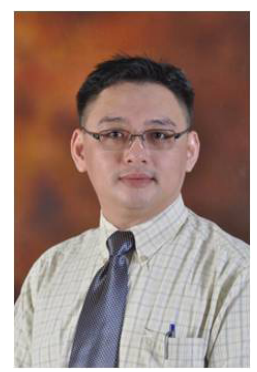

44)

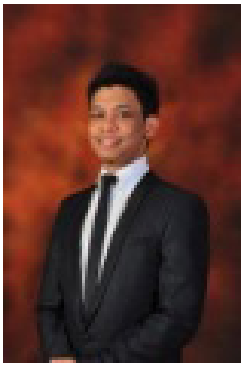

50)

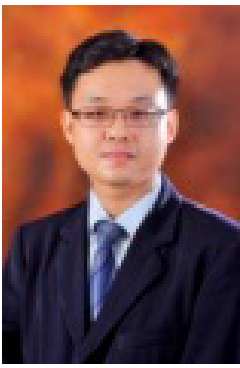

56)

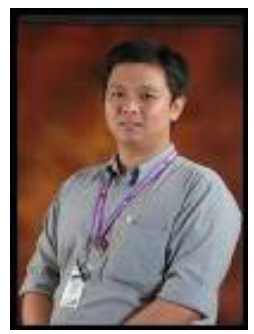

33)

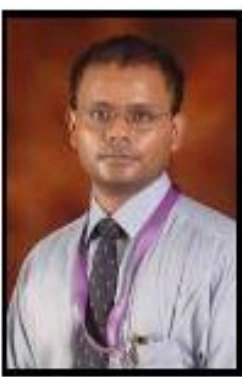

39)

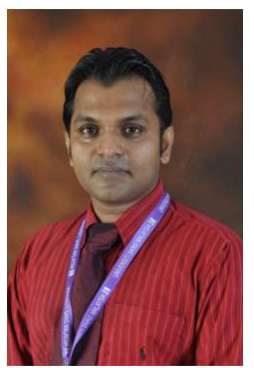

45)

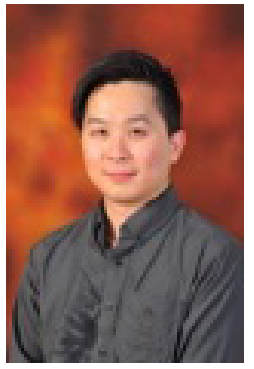

51)

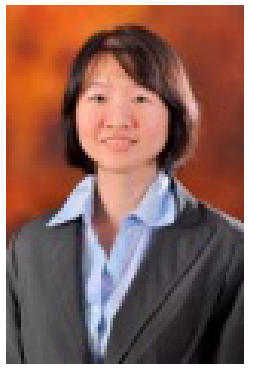

57)

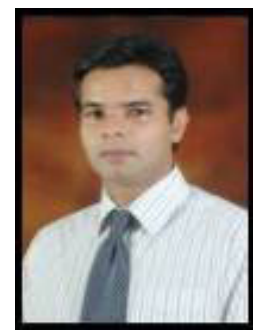

34)

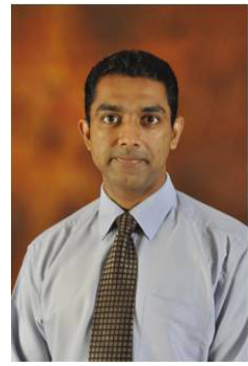

40)

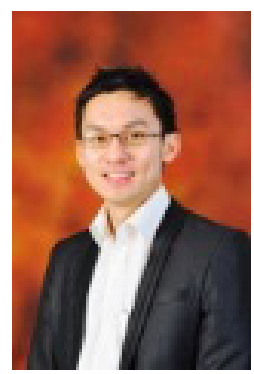

46)

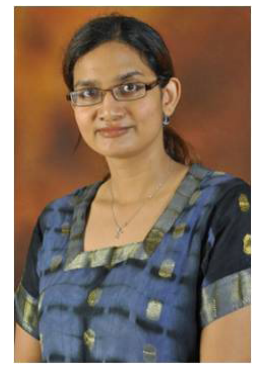

52)

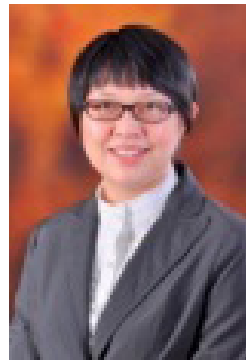

58)

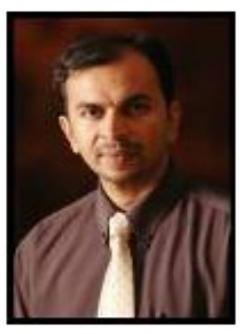

35)

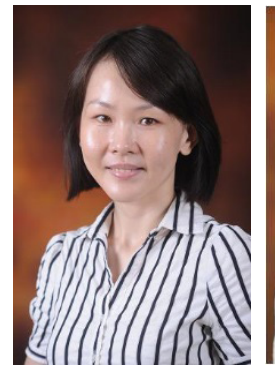

41)

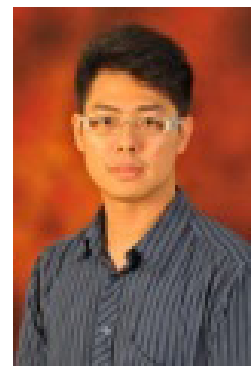

47)

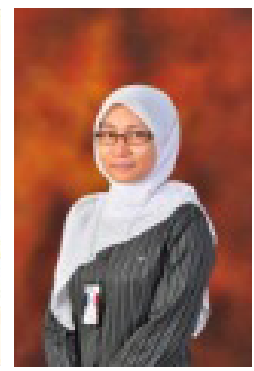

53）

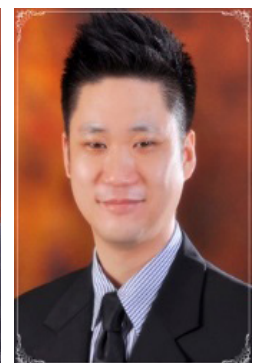

59)

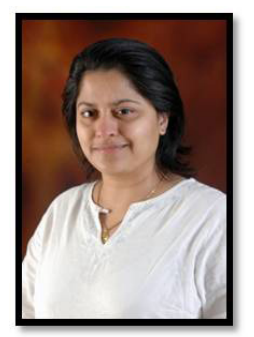

36)

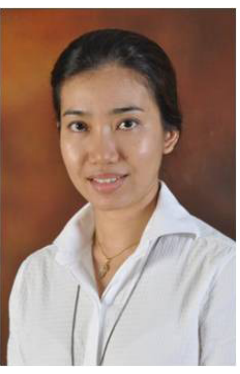

42)

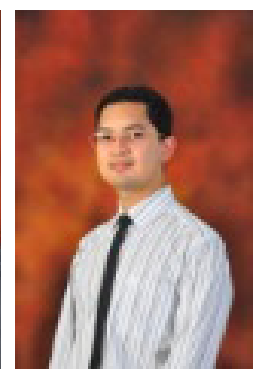

48)

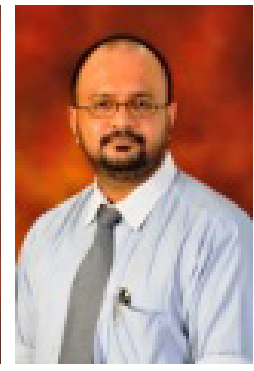

54)

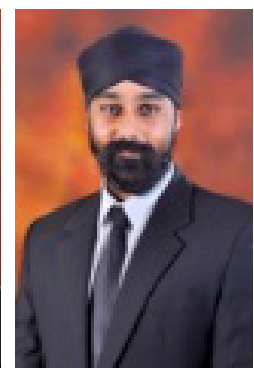

60) 
Editorial | Neurosciences Malaysia global precision brain health

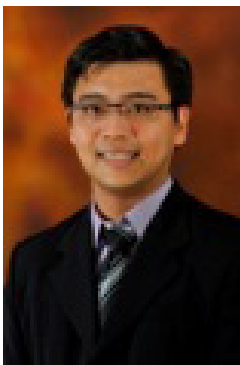

61)

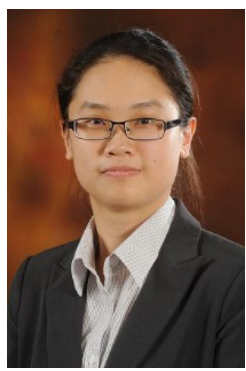

67)

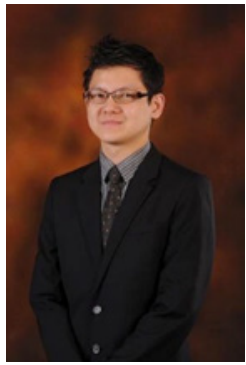

73)

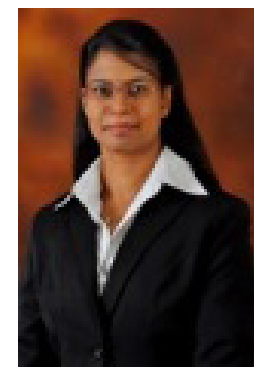

62)

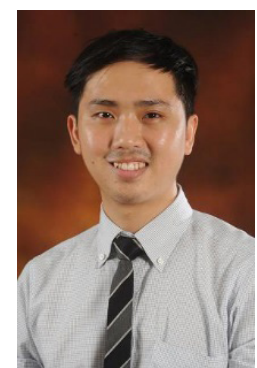

68)

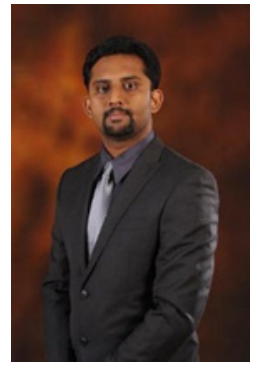

74)

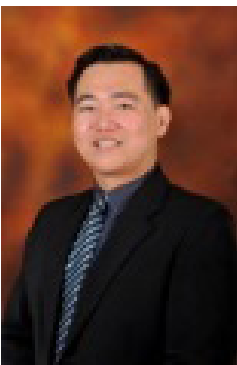

63)

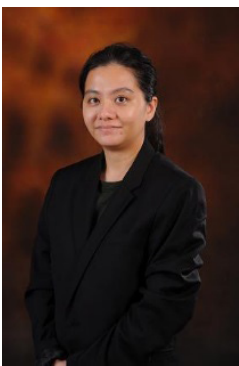

69)

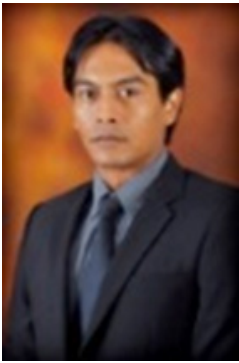

75)

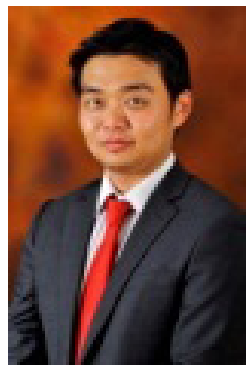

64)

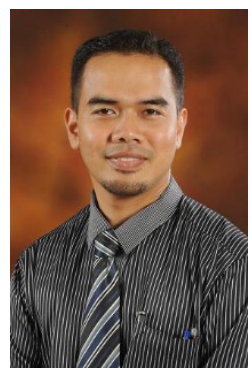

70)

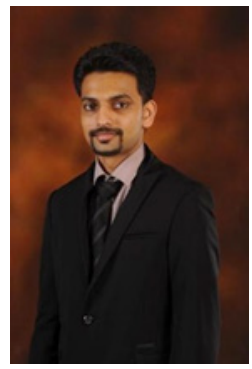

76)

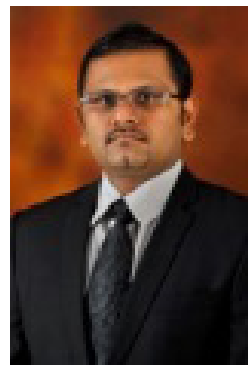

65)

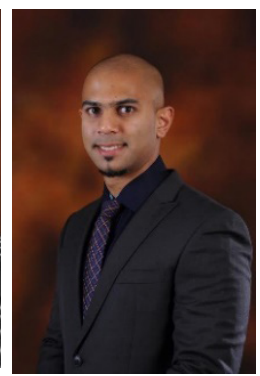

71)

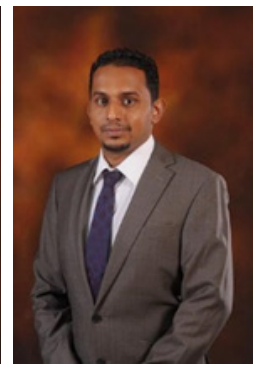

77)

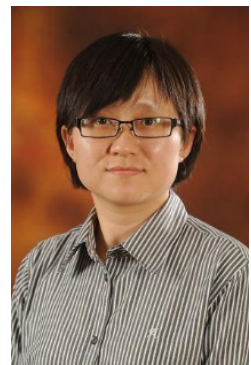

66)

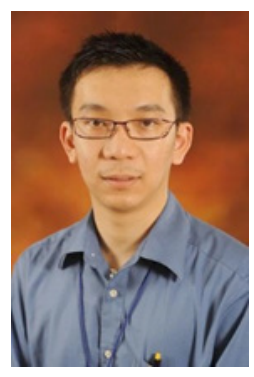

72)

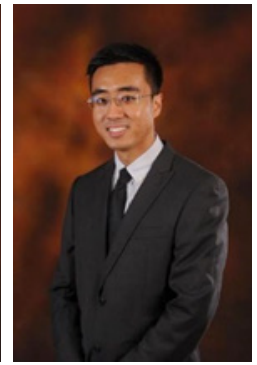

78)

Figure 2. Neurosurgeons who have graduated from the postgraduate neurosurgical programme since 2001

Remember your neurosurgical academic lineage: My neurosurgical teacher was Professor Luc Calliauw from Hospital University Ghent, Belgium who was taught by Professor Henk Verbiest from University Hospital Utrecht, Holland who was taught by Professor Clovis Vincent from Pitié-Salpêtrière Hospital, Paris, France who was taught by Professor Joseph Jules Francois Felix Babinski from the same hospital in France and the famous Professor Harvey Cushing of Harvard Medical School, USA
1) Professor Dr Zamzuri Idris
2) Associate Professor Dato' Dr Abdul Rahman Izaini Ghani
3) Dr Badrisyah Idris
4) Dr Adam Mohd Zakaria
5) Dr Kan Choon Hong
6) Dr Lee Hock Keong
7) Dr Gee Teak Shang
8) Dr Mohd Saufi Awang
9) Dr Farizal Fadzil
10) Dr Rahmat Harun @Haron
11) Dr Hafiz Mohd Zain
12) Dr Chan Kin Hup
13) Dr Sofan Zenian
14) Dr Saiful Razman
15) Dr Ahmad Zamzuri Remeli
16) Dr Mohd Aidil Mohd Noor
17) Dr Siti Suriyati Buang
18) Dr Sharon Casilda Theophilus
19) Dr Premananda Raja a/l Murugesa
20) Dr Sani Sayuthi 
21) Dr Saiful Azli

22) Dr Naseer Abdul Wahab

23) Dr Kantha a/l Rasalingam

24) Dr Toh Charng Jeng

25) Dr Tan Wei Ming

26) Dr Nujaimin Udin

27) Dr Mohd Azhari Omar

28) Dr Liew Boon Seng

29) Dr Adrean Husin

30) Dr Regunath a/l Kandasamy

31) Dr Risdhawati Hasan

32) Dr Asraf Sharifudin

33) Dr Lim Swee San

34) Dr Mohammad Azman Raffiq

35) Dr Gerard Arvind Martin

36) Dr Priya Sharda Jagdish Mitter

37) Dr Faizul Hizal Ghazali

38) Dr Puneet Nandrajog

39) Dr Kamalanathan a/l Palaniandy

40) Dr Thinesh Kumaran

41) Dr Cheah Pooi Pooi

42) Dr Siti Azleen Mohamad

43) Dr Ch'ng Chee How

44) Dr Sim Sze Kiat

45) Dr Ananda Arumugam

46) Dr Mah Jon Kooi

47) Dr Tan Yew Chin

48) Dr Mohd Raffiz Mohd Ali

49) Dr Jason Raj a/l Johnson Kovilpillai

50) Dr Fadzlishah Johanabas Rosli
51) Dr Adrian Ng Wei Chih

52) Dr Jacintha Vikeneswary

53) Dr Ailani Ab Ghani

54) Dr Senthil Kumar a/l Rajapathy

55) Dr Ng Wei Peng

56) Dr Ariz Chong Abdullah @ Chong Chee Yong

57) Dr Low Siaw Nee

58) Dr Low Yong Lee

59) Dr Lim Liang Hooi

60) Dr Manvinder Singh Mangat

61) Dr Lai Chuang Chee

62) Dr Kanmani Dewi

63) Dr Chan Chee Kong

64) Dr Mohd Syahiran bin Mohd Sidek

65) Dr Prabu Rau a/l Siram

66) Dr Low Peh Hueh

67) Dr You Xinli

68) Dr Goh Chin Hwee

69) Dr Neoh Yee Yik

70) Dr Muhammad Ihfaz Ismail

71) Dr Davendran Kanesen

72) Dr Kho Giat Seng

73) Dr Nelson Yap Kok Bing

74) Dr Rajendra Rao a/l Ramalu

75) Dr Rakesh Rethinasamy

76) Dr Ramissh Paramasivam

77) Dr Vinodh a/l Vayara Perumall

78) Dr Yee Sze Voon 
Editorial | Neurosciences Malaysia global precision brain health

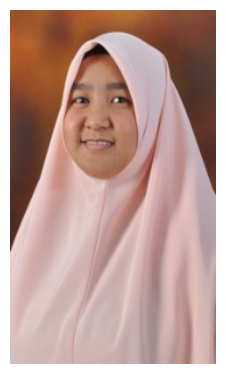

1)

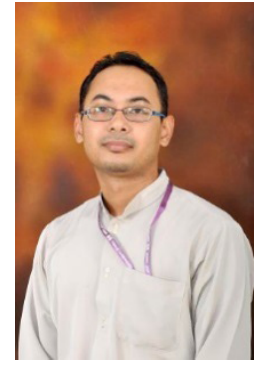

7)

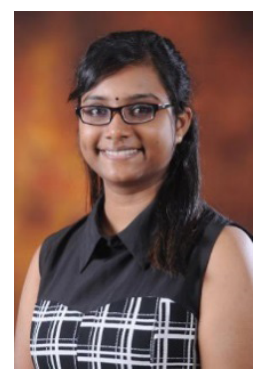

13)

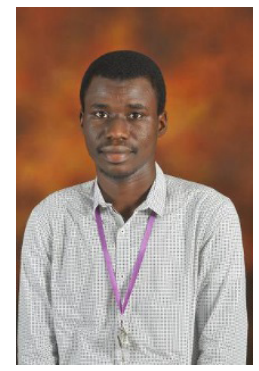

19)

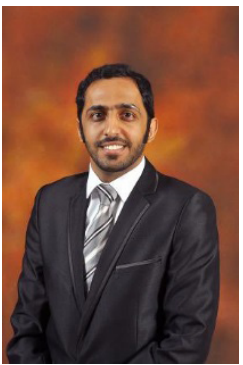

25)

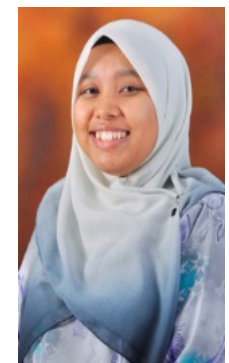

2)

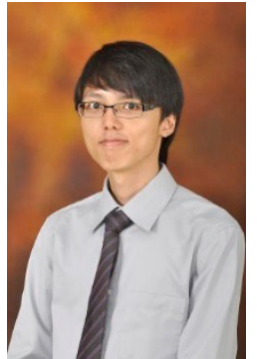

8)

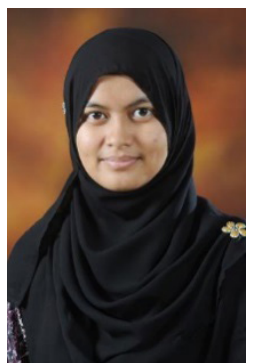

14)

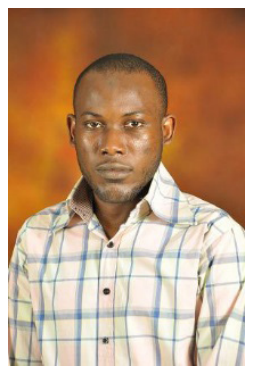

20)

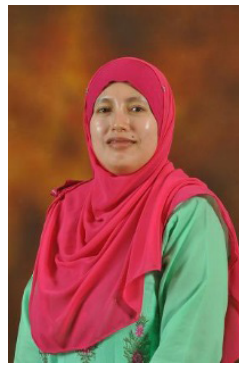

26)

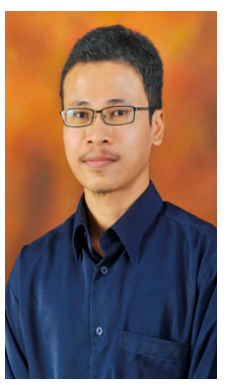

3)

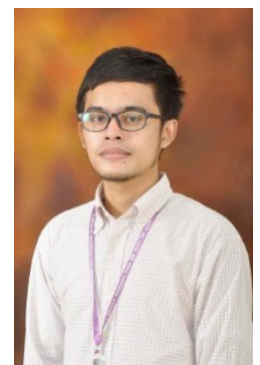

9)

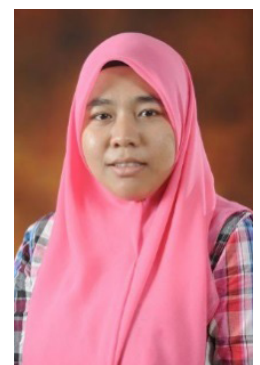

15)

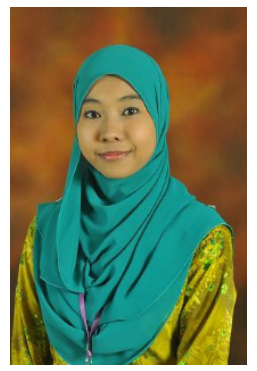

21)

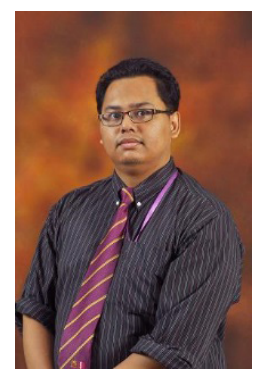

27)

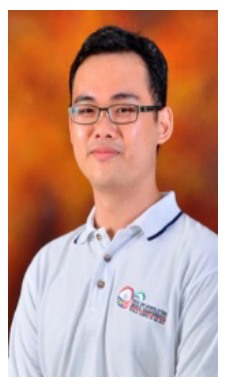

4)

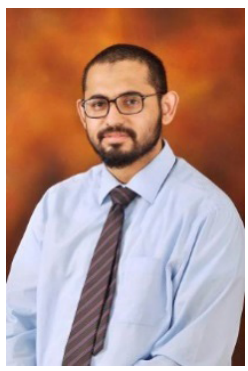

10)

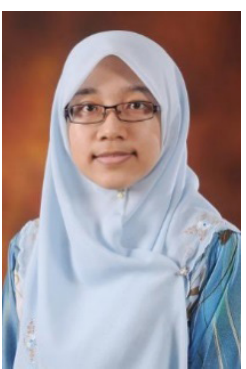

16)

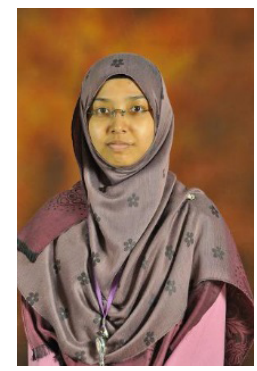

22)

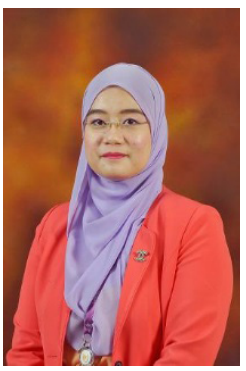

28)

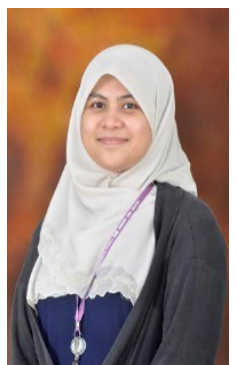

5)

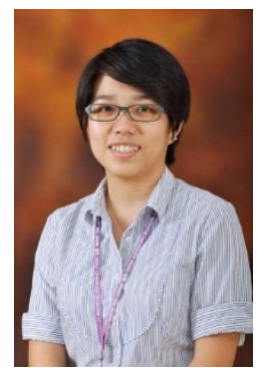

11)

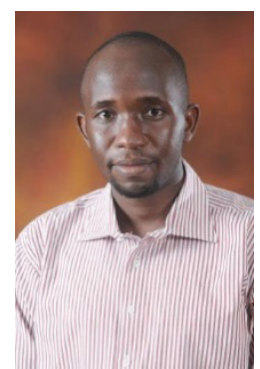

17)

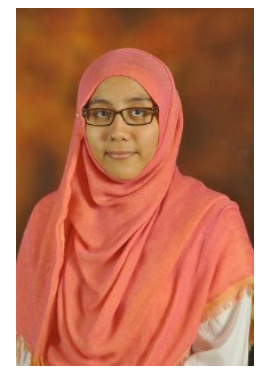

23)

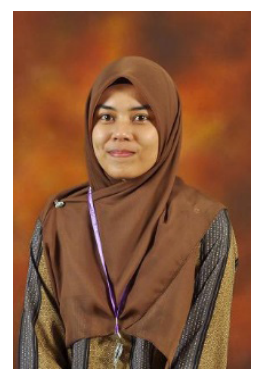

29)

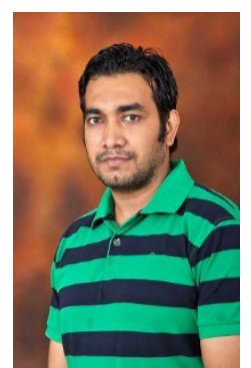

6)

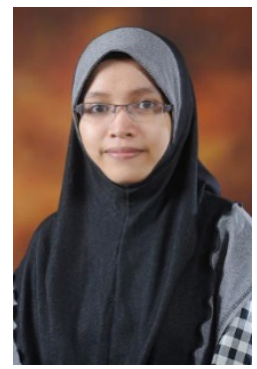

12)

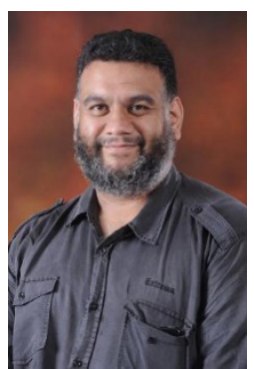

18)

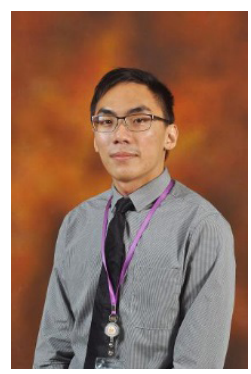

24)

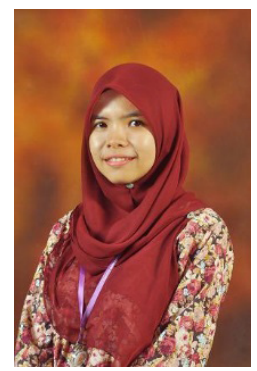

30) 


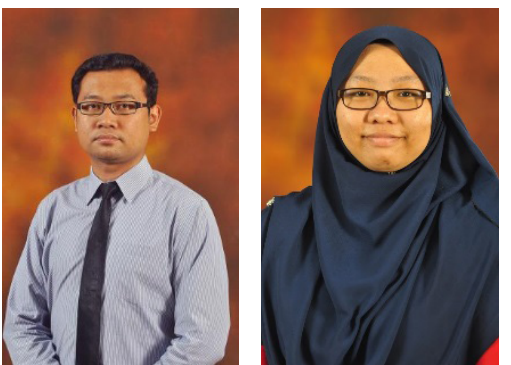

31)

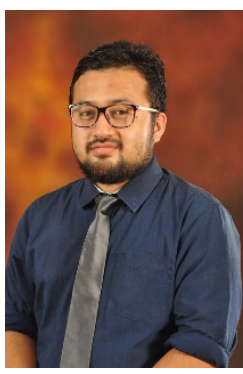

37)

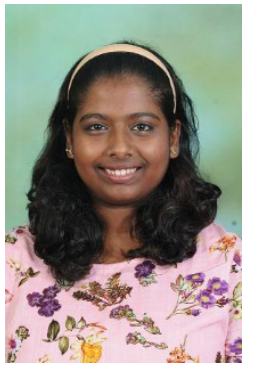

43)

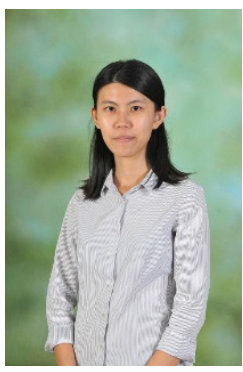

49)

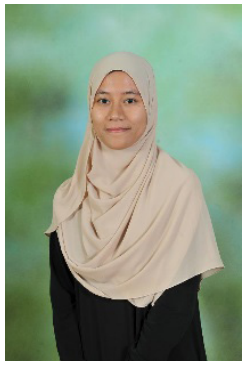

55)
32)

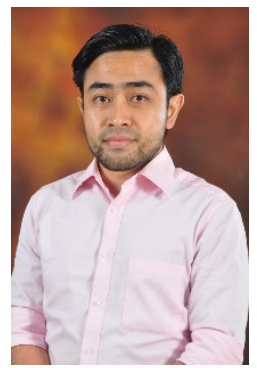

38)

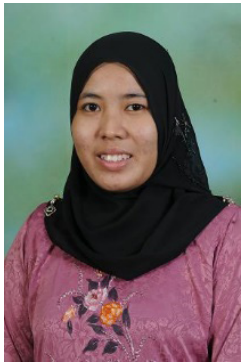

44)

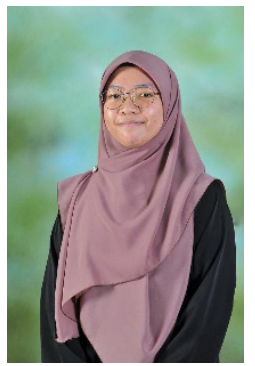

50)

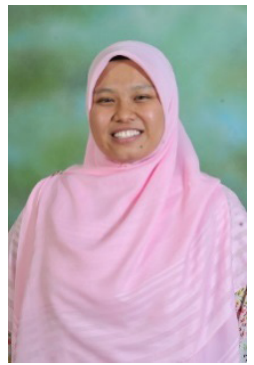

56)

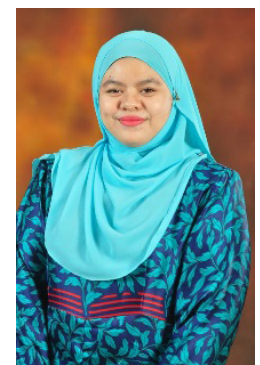

33)

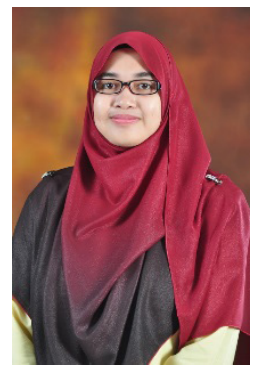

39)

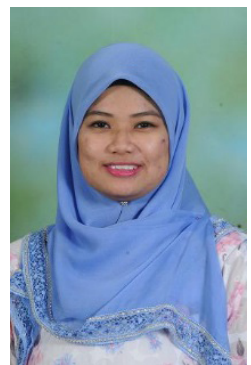

45)

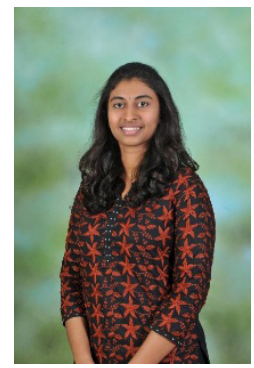

51)

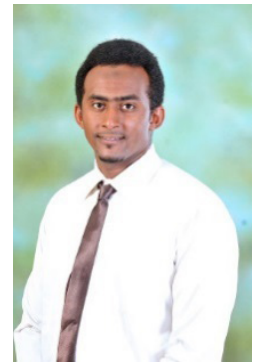

57)

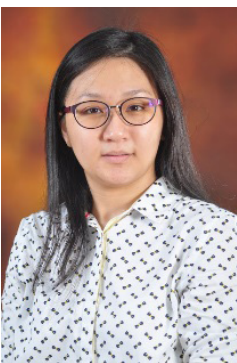

34)

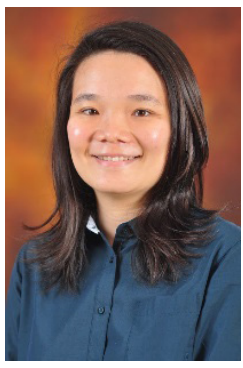

40)

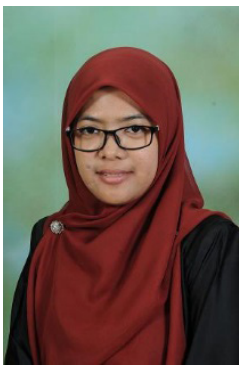

46)

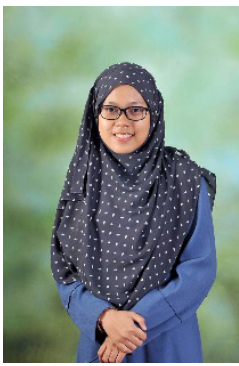

52)

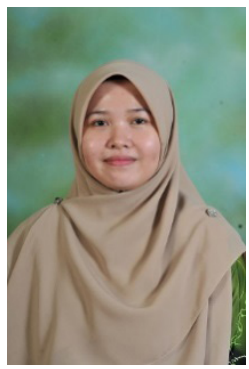

58)

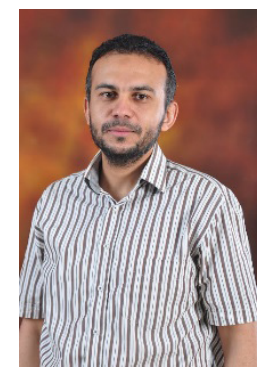

35)

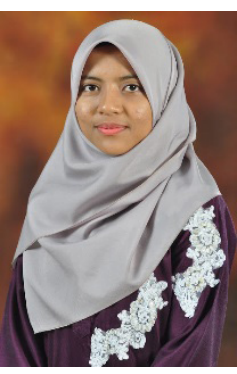

41)

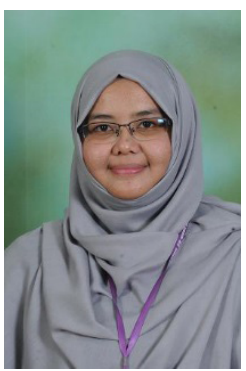

47)

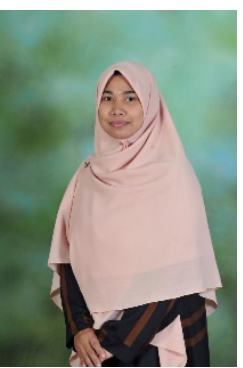

53)

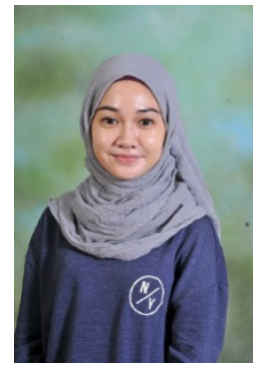

59)

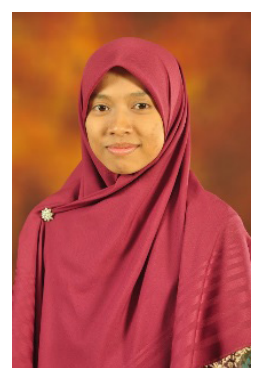

36)

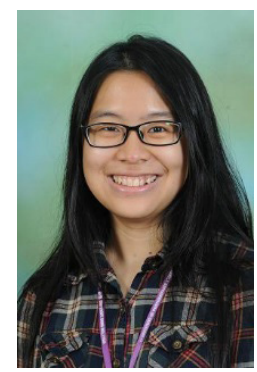

42)

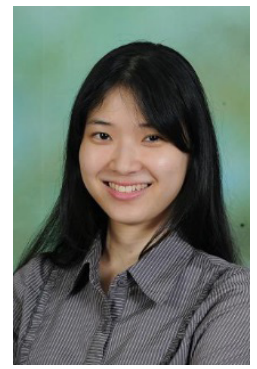

48)

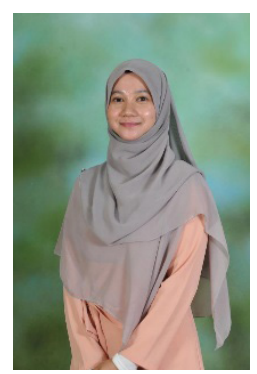

54)

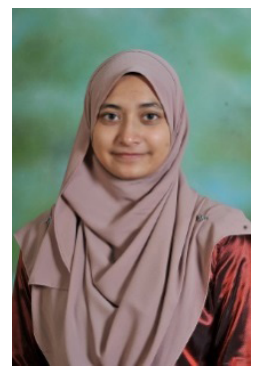

60) 
Editorial | Neurosciences Malaysia global precision brain health

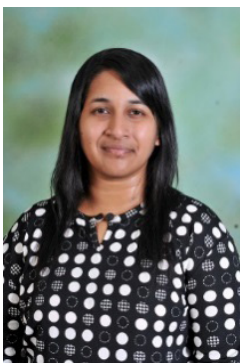

61)

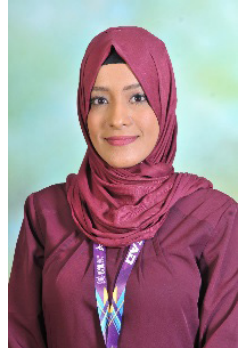

67)

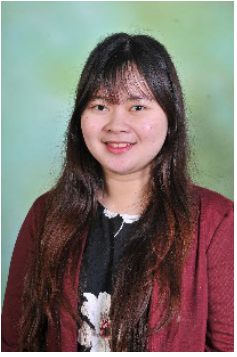

73)

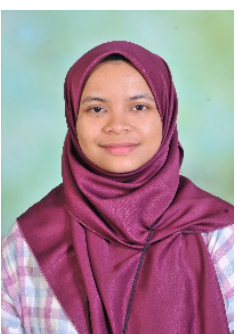

79)

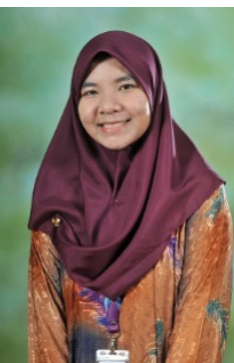

63)

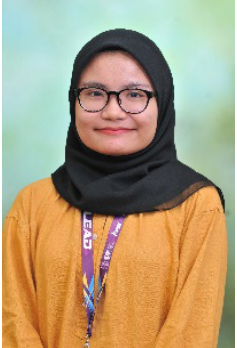

68)

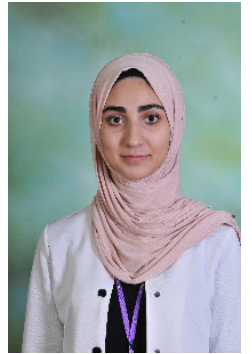

74)

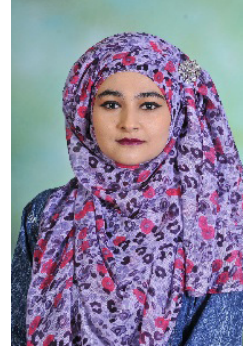

69)

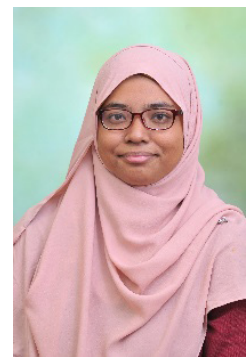

75)

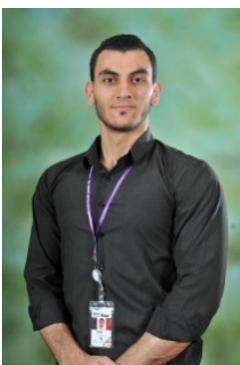

64)

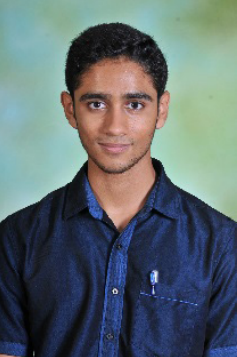

70)

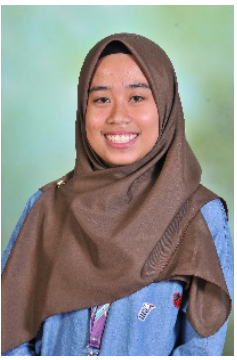

76)

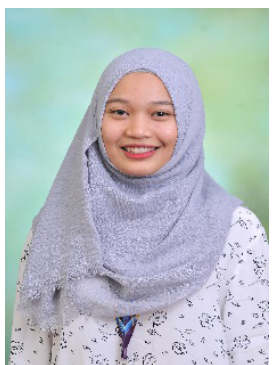

65)

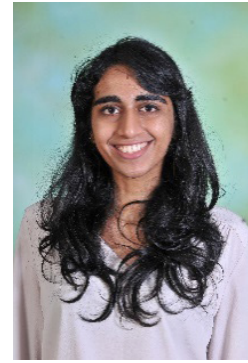

71)

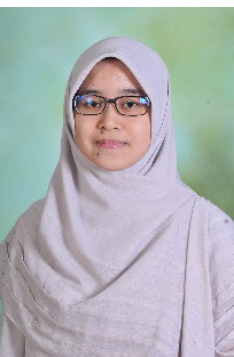

77)

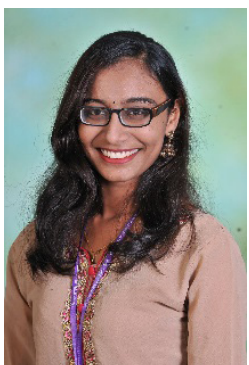

66)

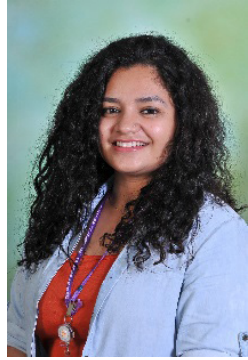

72)

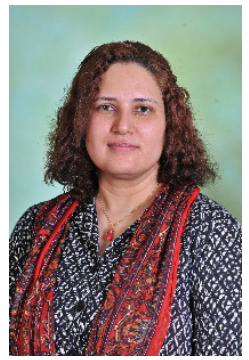

78)

Figure 3. Integrated neuroscience programme students from 1st batch till 11th batch

1st batch INP students

1) Dr.Wan Mohd Daud Wan Omar

2) Nazirah Hanim Sharipudin

3) Rumaisa Abu Hassan

4) Teo Yong Chang 2nd batch INP students

5) Al-Zahrani Hussain Saad

6) Che Mohd Nasril Che Mohd Nasir

7) Koh Junhao

8) Mohd Ibrahim Abdullah

9) Muhammad Bilal

10) Nazeerah Abd Rahman

11) Wong Jia Hui 
3rd batch INP students

12) Saidah Napisah Muhammad

13) Priatharsine Seerangan

14) Nurul Fatihah Othman

15) Nur Aimi Zawami Ahmad

16) Fatin Hilyani Mohamad

17) Ahmad Adamu Adamu

18) Usman Jaffer 4th batch INP students

19) Abu Bakar Tijjani Salihu

20) Auwal Bello Hassan

21) Norlyiana Samsuri

22) Rafidah Rosli

23) Siti Nur Ain Zakaria $5^{\text {th batch INP students }}$

24) Chuang Huei Gau

25) Faraj Almarri

26) Mazira Mohamad Ghazali

27) Muhammad Afiq Mahayidin

28) Nor Aqilah Mohd Yusuf Yeo

29) Nurfaizatul Aisyah Ab Aziz

30) Nurul Atikah M Nor Nazli

31) Shazlan Noor Suhaimi 6th batch INP students

32) Amanina Ahmad Safri

33) Amy Shafinas Azman

34) Chong Pei Nei

35) Khaled Sayed Rabia Elsayed

36) Mas Syazwanee Shab

37) Mohd Khairul Izamil Zolkefley

38) Mohd Waqiyuddin Abdullah

39) Nadia Izzati Nordin

40) Tai Yan Shan

41) Ummi Nasrah Talib

7th batch INP students

42) Chai Wen Jia

43) Ivanna Fernandez

44) Nur Adila Ramli
45) Nur Naznee Hirni Abd Aziz

46) Nurfarhana Abdul Ghani

47) Siti Atiyah Ali

48) Tan Celina

8th batch INP students

49) Cheeh Hui Lee

50) Dayang Yasmin Abang Abdul

51) Revathy Murali

52) Siti Hajar Mohd Zaki

53) Siti Nurulhusna Hashim

54) Tiara Ramli

55) Zakiyyah Munirah Mohd Zaki 9th batch INP students

56) Aishah Sakinah Zahid

57) Mohammed Abdalla Kannan Ahmed

58) Nor Azulaikha Abdullah

59) Nurul Nazihah Zaidil

60) Sara Fatini Abdul Naser

61) Sri Ratha Balakrishnan 10th batch INP students

62) Farah Madihah Mustafa Kamal

63) Nurul Amirah Mohammad Murad

64) Younis MS Firwana 11th batch INP students

65) Anis Raihan Dzeidee Schaff

66) Devi AP Ananth

67) Hadjer Khiati

68) Iffah Syafiqah Suhaili

69) Iman Imtiyaz Ahmed Juvale

70) Joshua Kuruvilla

71) Kiirtaara Aravindhan

72) Malvika Sharma

73) Nornadirah Abdul Rahman

74) Nour Mohammed Abdallah Qaddumi

75) Nur Nasihken Mawi

76) Nurdarina Ausi Zulkifli

77) Nurulain Syahirah Razali

78) Rabia Nazir

79) Wan Shahirah Wan Adnan 
Editorial | Neurosciences Malaysia global precision brain health

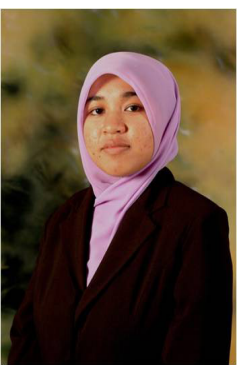

1)

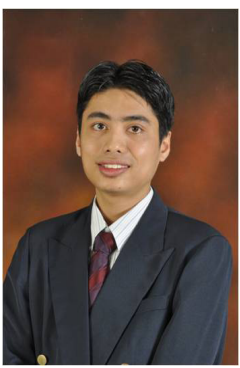

7)

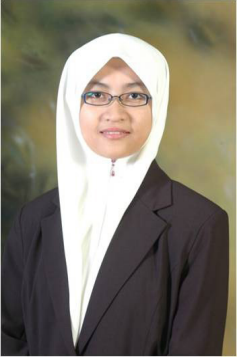

13)

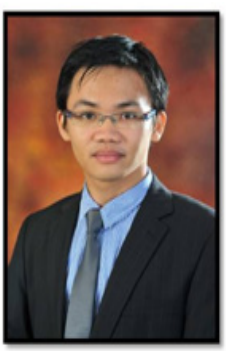

19)

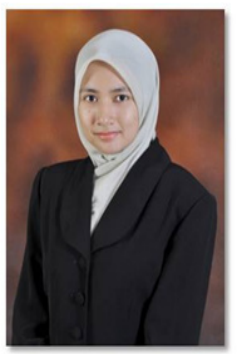

25)

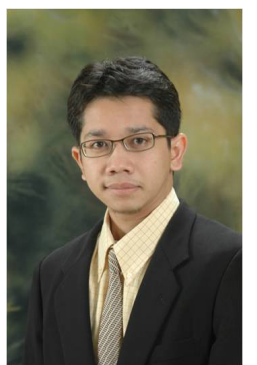

2)

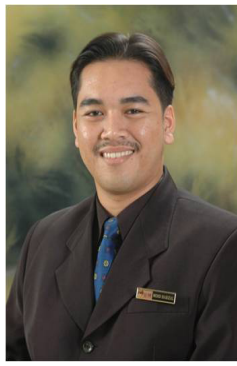

8)

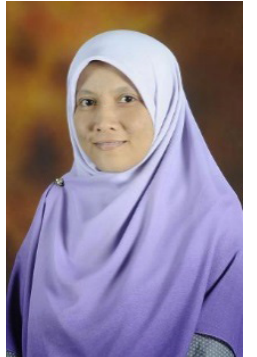

14)

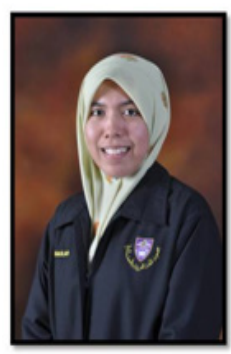

20)

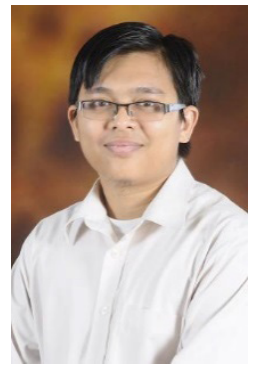

26)

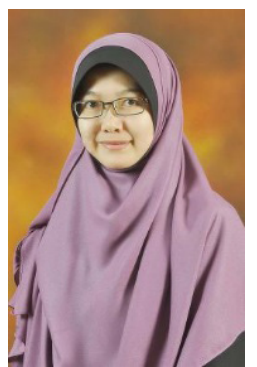

3)

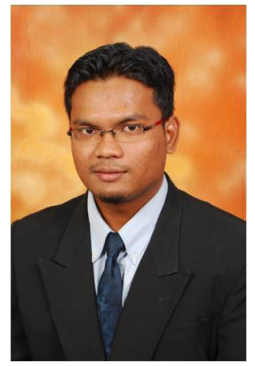

9)

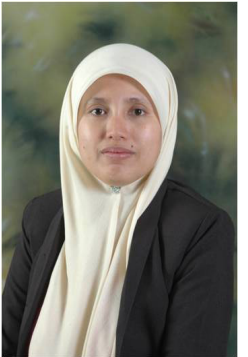

15)

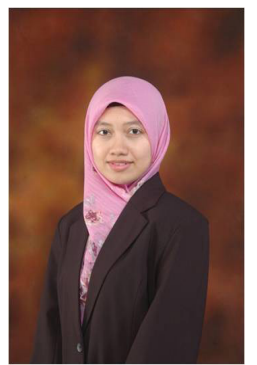

21)

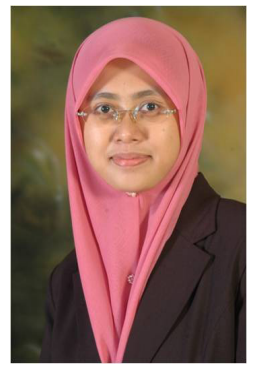

27)

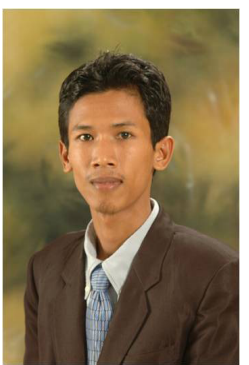

4)

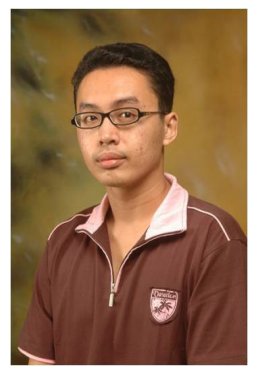

10)

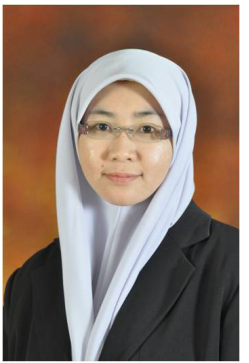

16)

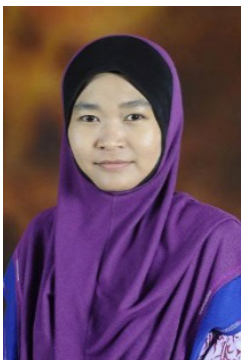

22)

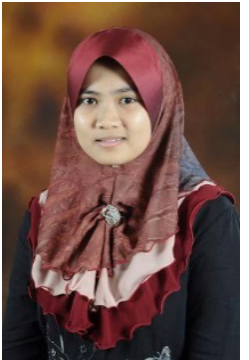

28)

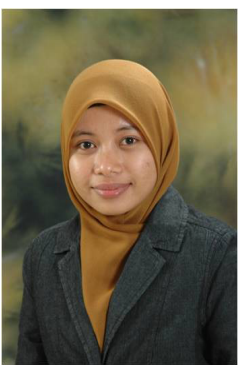

5)

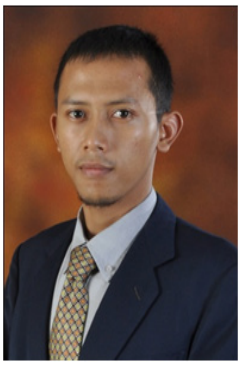

11)

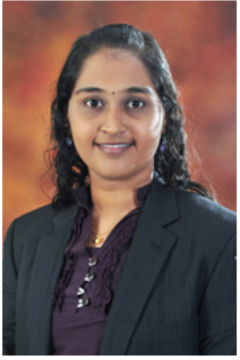

17)

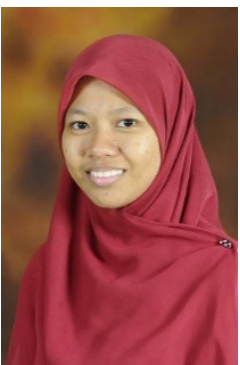

23)

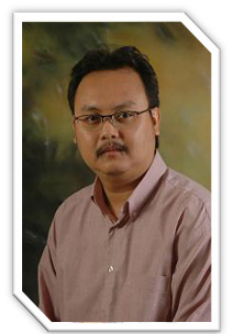

29)

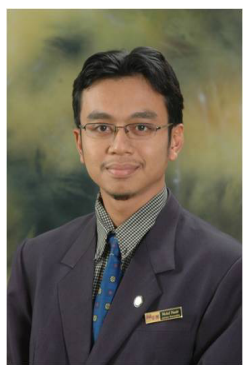

6)

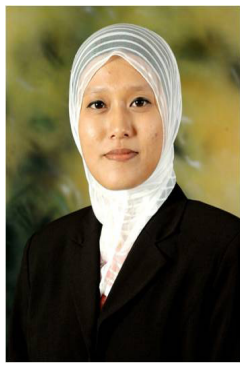

12)

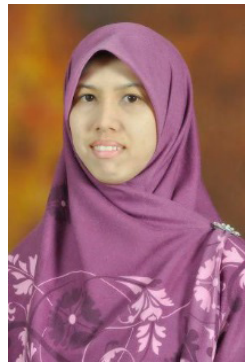

18)

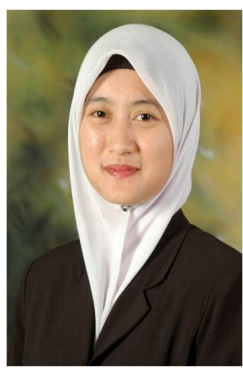

24)

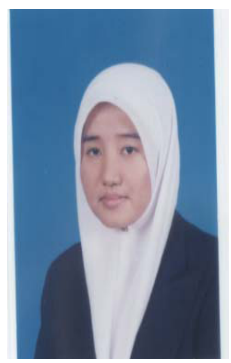

30) 


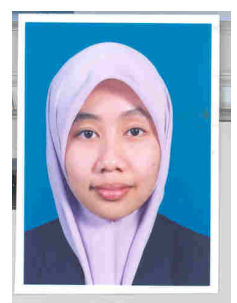

31)

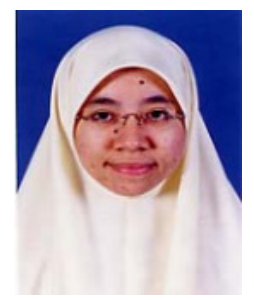

32)

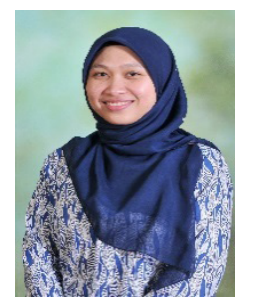

33)

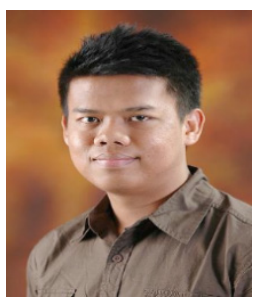

34)

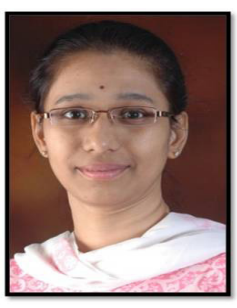

35)

Figure 4. Master by Pure Research, Doctorate by Pure Research and Advanced Master of Medicine (Neurology)

Neurosciences
1) Siti Norhajah Hashim
2) Mohd Azim Patar
3) Putri Nur Hidayah Al-Zikri Mohamad Akil
4) Dr. Zulkifli Mustafa
5) Raisah Abd Hadi
6) Mohd Nasir Mat Nor
7) Syed Fariq Fathullah Syed Yaacob
8) Mohd Harizal Senik @ Nawi
9) Mohd 'Ulul ‘Ilmie Ahmad Nazri
10) Ahmad Tarmizi Che Has
11) Muhammad Hanif Che Lah
12) Norshazrin Shazira Shafee
13) Siti Zawani Mohd Ramli
14) Wan Noor Ainun Baharuddin
15) Mazira Mohamad Ghazali
16) Samhani Ismail
17) Nanthini a/p Jayabalan
18) Wan Salihah Wan Abdullah

19) Tee Jong Huat

20) Khalilah Haris

21) Khairol Naaim Mohd Nasir

22) Nur Syairah Abd Rani

23) Mashytah Abdul Karim

24) Nor Entan Supeno

25) Nurul Iman Wan Ismail

26) Asyraf Abd Rahman

27) Emmilia Husni Tan

28) Siti Zulaikha Nashwa Mohd Khair

29) Abdul Aziz Mohamed Yusoff

30) Farizan Ahmad

31) Norafiza Zainudin

32) Sarina Sulong

33) Siti Naziha Hasma Hassan

34) Zarif Sofian

Neurology

35) Dr Shalini Bhaskar 
Editorial | Neurosciences Malaysia global precision brain health

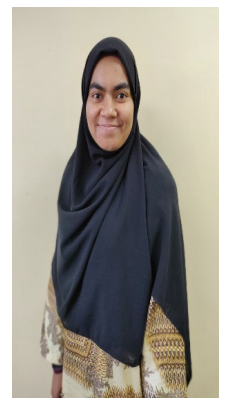

1)

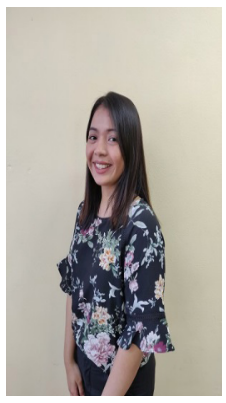

7)

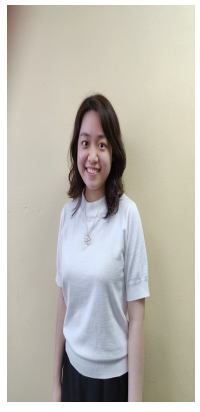

13)

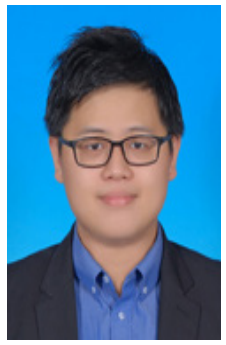

19)

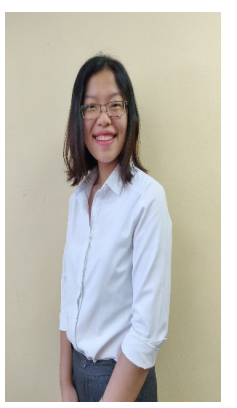

2)

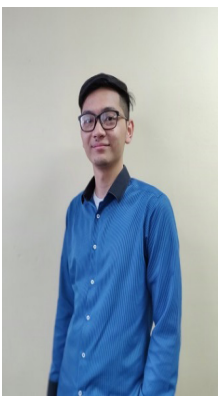

8)

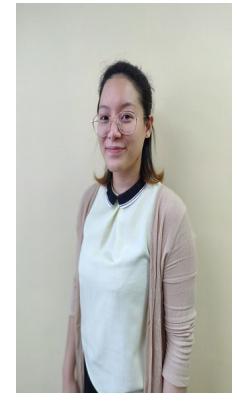

14)

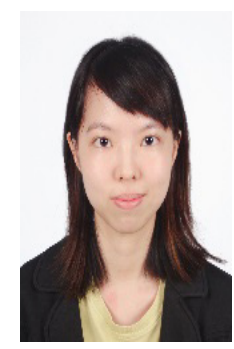

20)

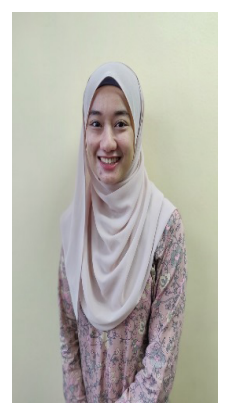

3)

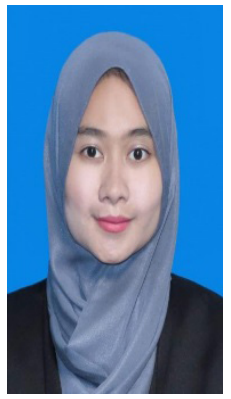

9)

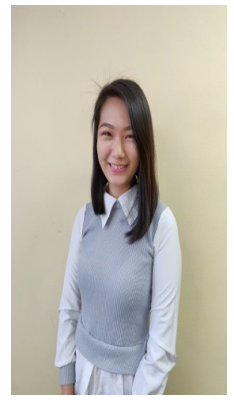

15)

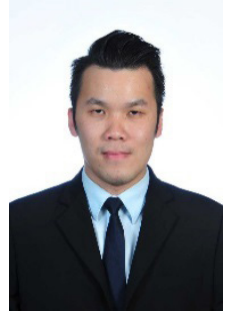

21)

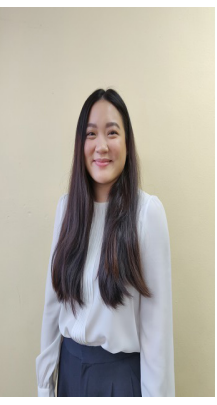

4)

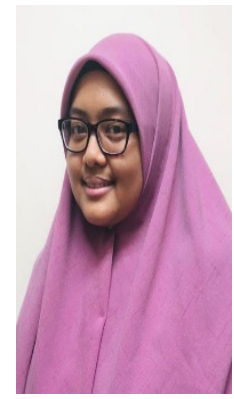

10)

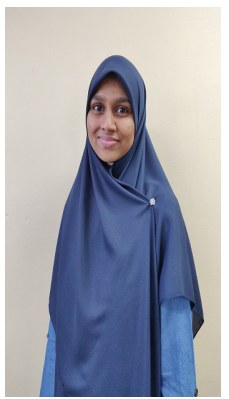

16)

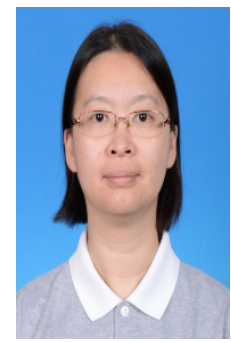

22)

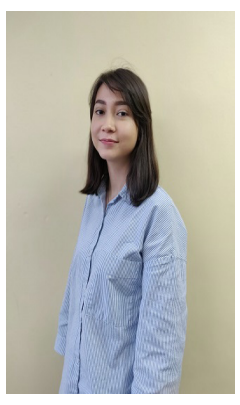

5)

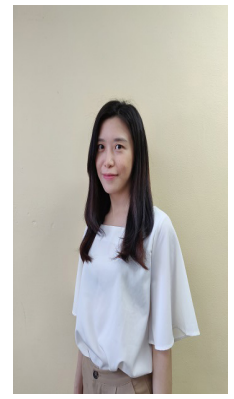

11)

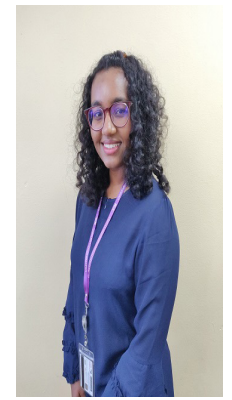

17)

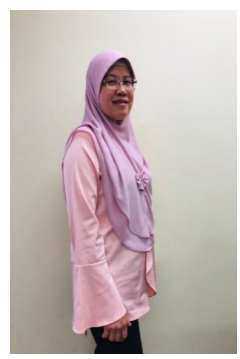

23)

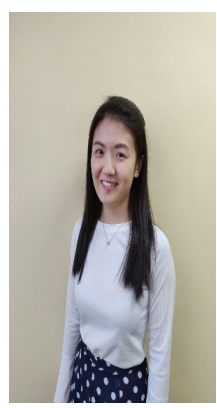

6)

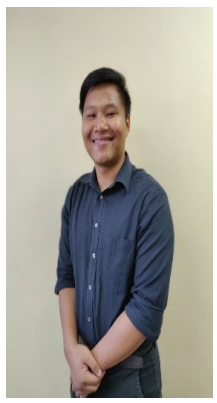

12)

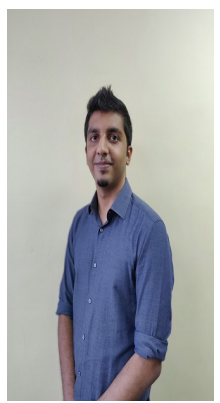

18)

Figure 5. First batch Master of Clinical Psychology students USM-UPSI

1st batch Master Integrated Psychology Programme-USM
1) Ummi Norliyana Zainal
2) Angela Chen Aun Kei
3) Siti Amirah Hanna Jafri Malin

4) Audrey Poh Sze Huey

5) Sharifah Diana Syed Abdul Halim

6) Loh Ken Joey

7) Mariah Hanna

8) Mohamad Farahan Huszaimi M Pajar 
9) Norsuhana Emilinadiah Husin

10) Nurulhidayah Mohd Saat

11) Seeh Ti Whan

1st batch Master Integrated Psychology Programme-UPSI

12) Muhammad Lutfi Md Nizam

13) Neo Jia Yeh

14) Norasyikin Jane Mustafa Kamal

15) Chao Thung Yin (Joyce)

16) Asmirah Jafarulla Khan

17) Suwarna Sukumaran

18) Abd Raouf Abdul Razak 1st batch Doctorate Integrated Psychology Programme-USM

19) Tay Kok Wai (Clinical Neuropsychology)

20) Michelle Choong Poh Kin (Clinical Psychology)

21) Liang Yaw Wen (Clinical Psychology)

1st batch Doctorate Integrated Psychology Programme-UPSI

22) See Geok Lan (Clinical Neuropsychology)

23) Atiqah Chew Abdullah (Clinical Psychology) 
Editorial | Neurosciences Malaysia global precision brain health

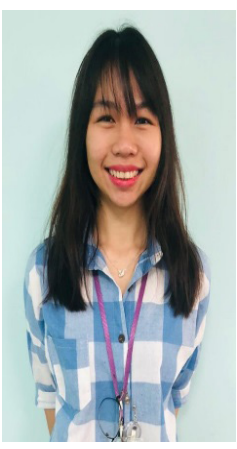

1)

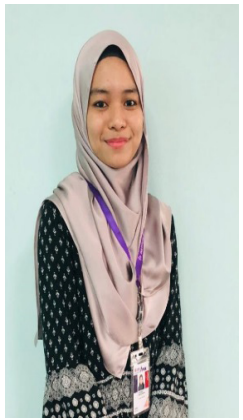

7)

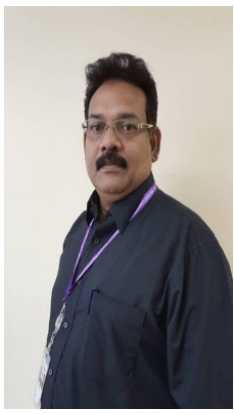

13)

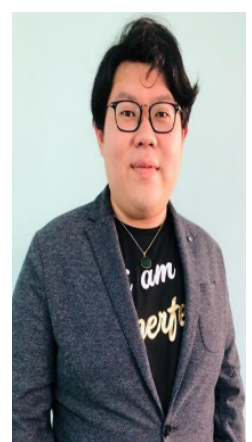

2)

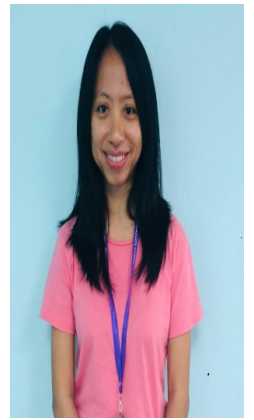

8)

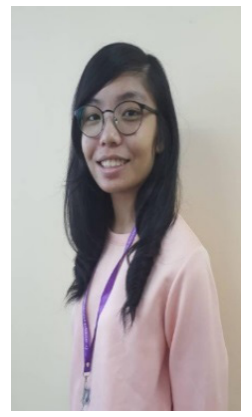

14)

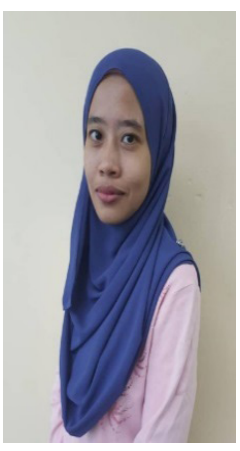

3)

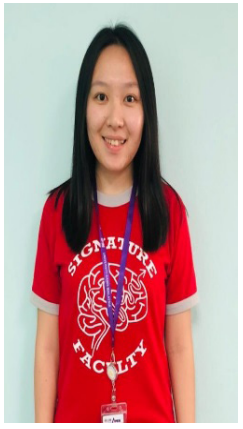

9)

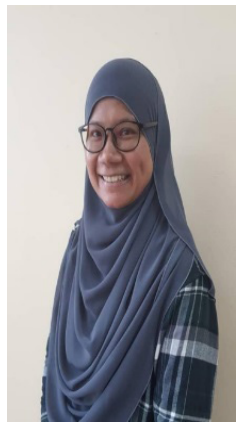

15)

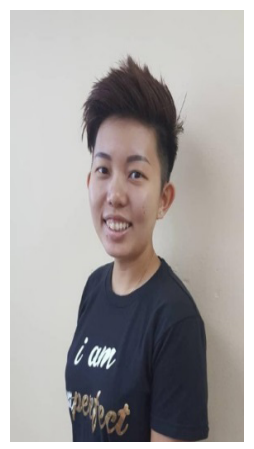

4)

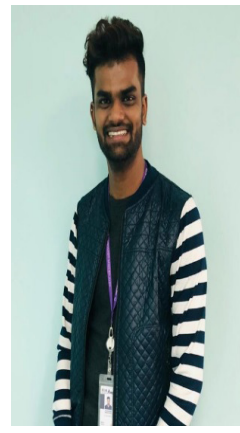

10)

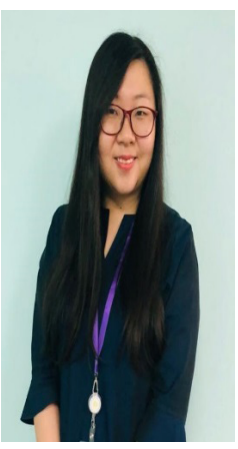

5)

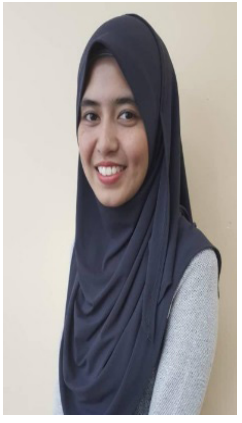

11)

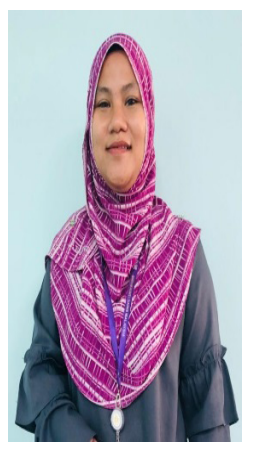

6)

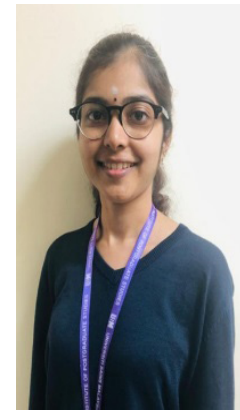

12)

Figure 6. First and second batch of Master of Cognitive Neurosciences USM offered at Postgraduate Institute@Kuala Lumpur

1st batch

1) Joice

2) Wang Shi-Jie

3) Nurul Bayti Sumardi

4) June Lew Wan Ling

5) Chin Jia Wei

6) Norrul Aikma Mohamed

7) Khairiah Razali

8) Kartina Ismail

9) Lim Hui Sean
10) Yogendren Murugia

11) Nadiah Mohd Sukarno

2nd batch

12) Thilageswary a/p Doraisamy

13) Ramesh Kumar a/l Ramachandren

14) Audrey Antoine a/p Max Antoine

15) Eizan Azira Mat Sharif 


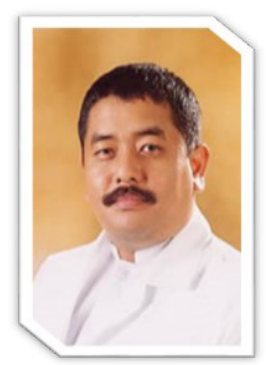

1)

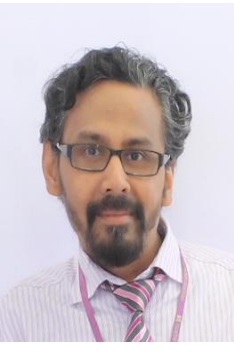

7)

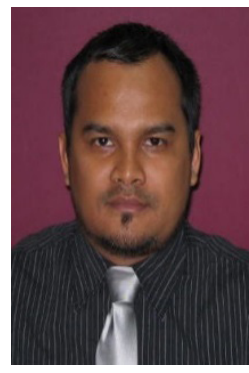

13)

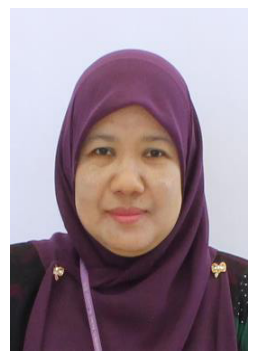

19)

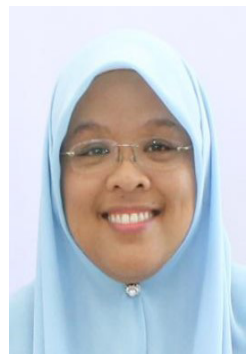

25)

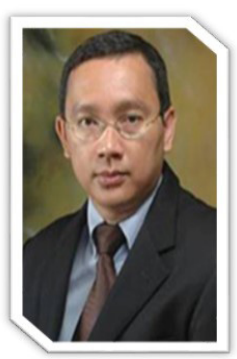

2)

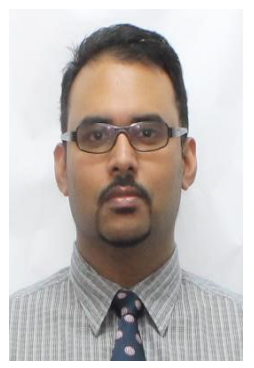

8)

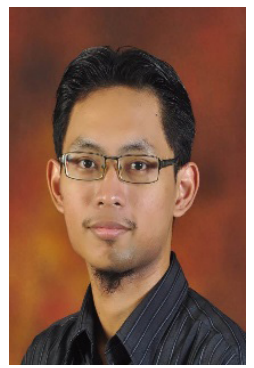

14)

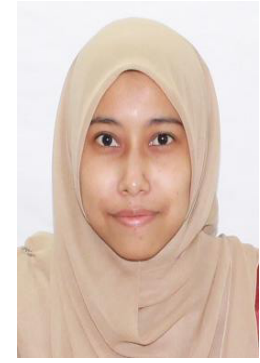

20)

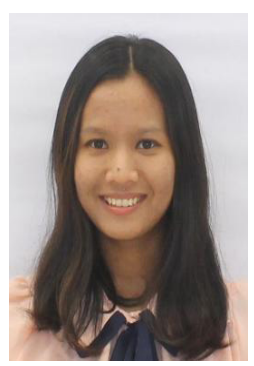

26)

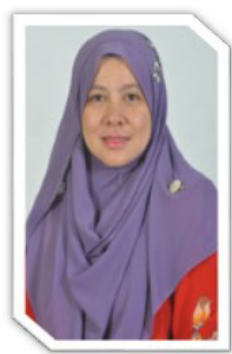

3)

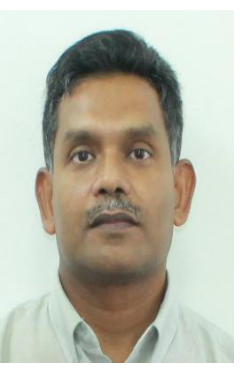

9)

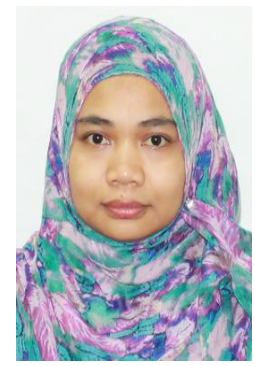

15)

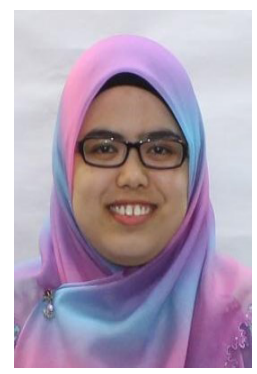

21)

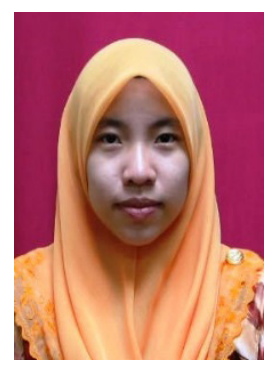

27)

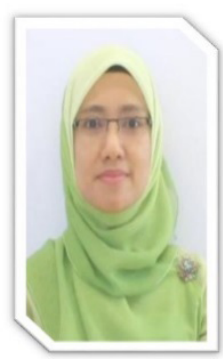

4)

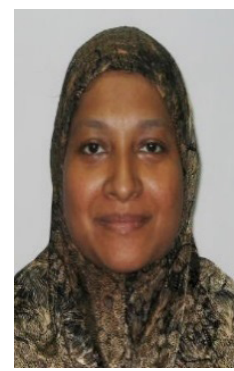

10)

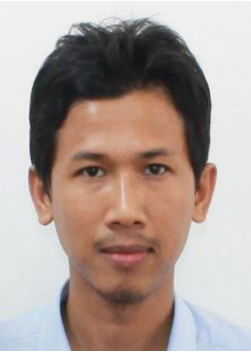

16)

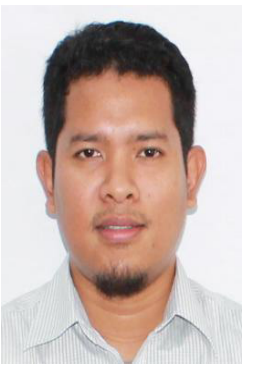

22)

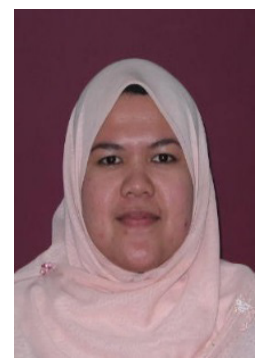

28)

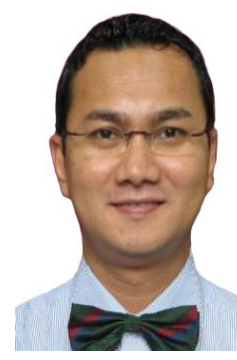

5)

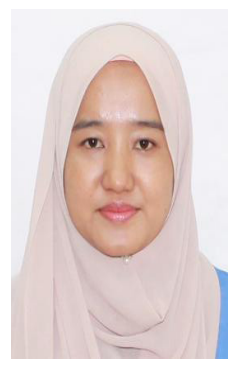

11)

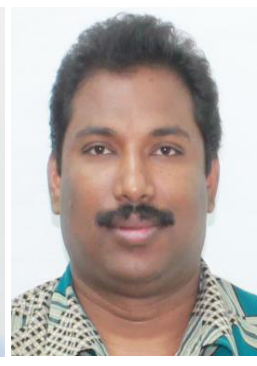

17)

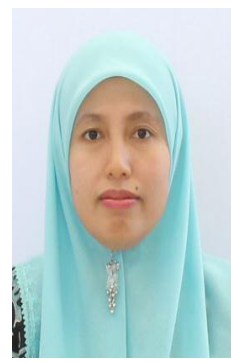

23)

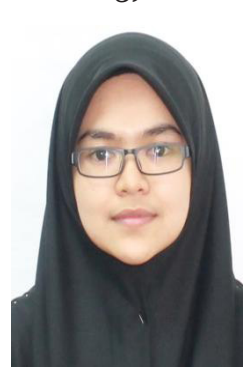

29)

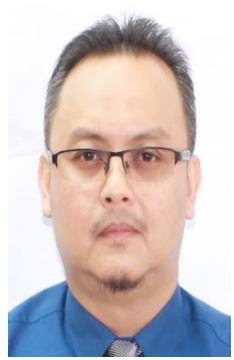

6)

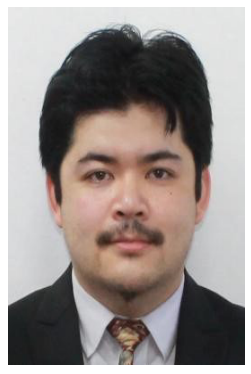

12)

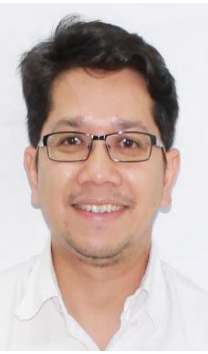

18)

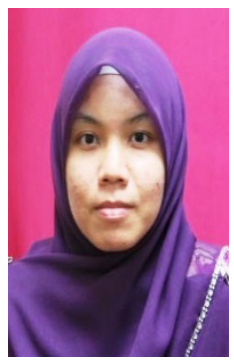

24）

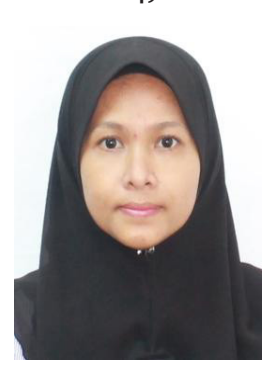

30) 
Editorial | Neurosciences Malaysia global precision brain health

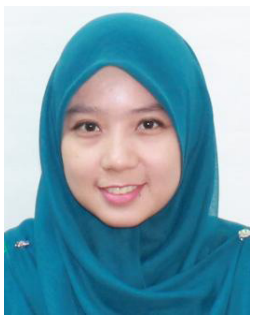

31)

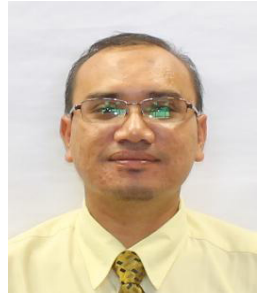

37)

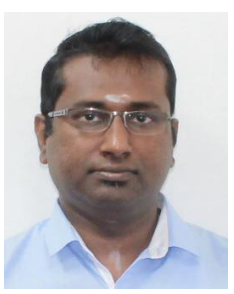

43)

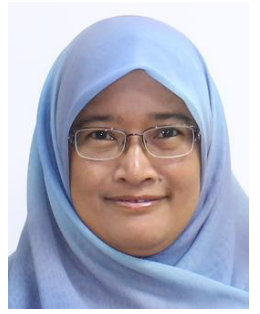

49)

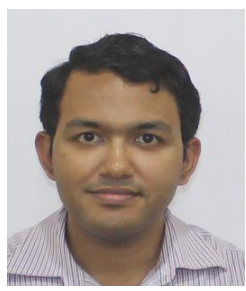

55)

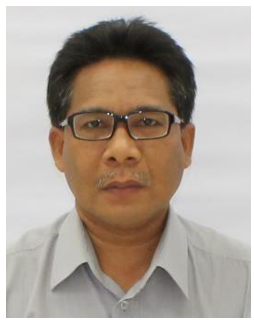

32)

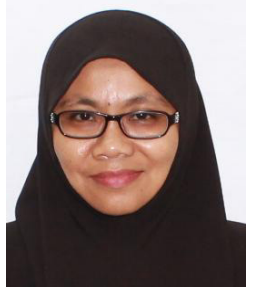

38)

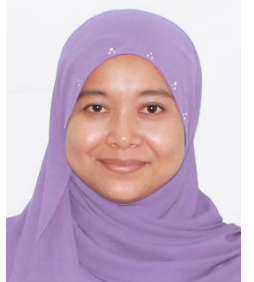

44)

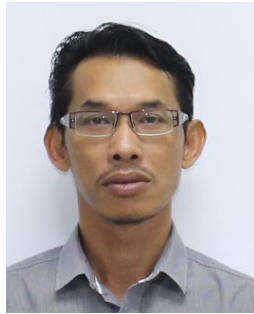

50)

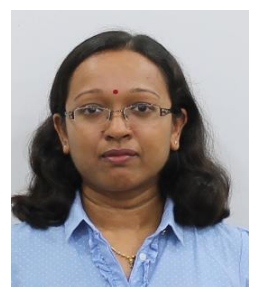

56)

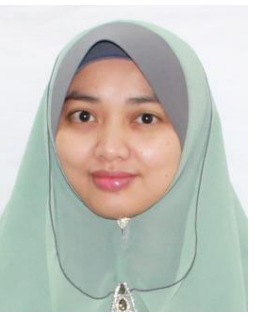

33)

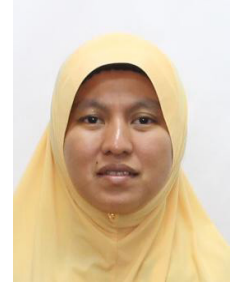

39)

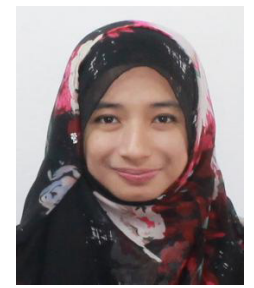

45)

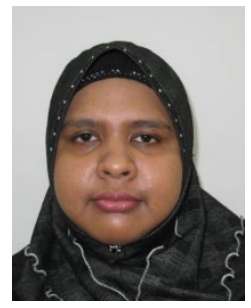

51)

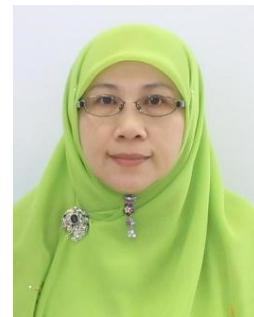

34)

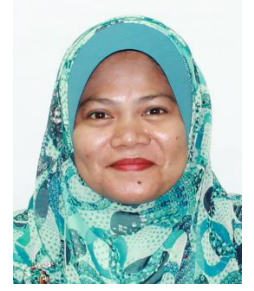

40)

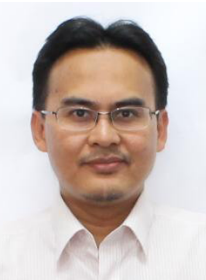

46)

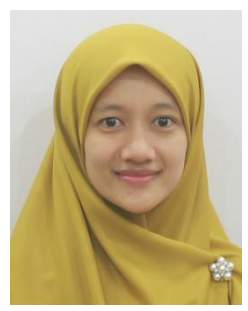

52)

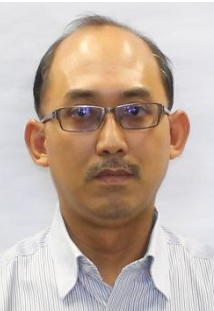

35)

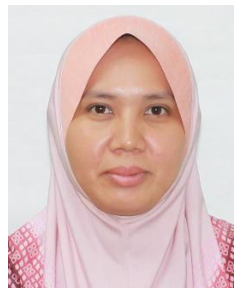

41)

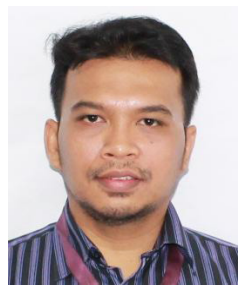

47)

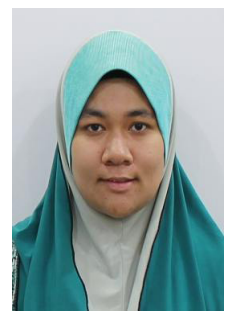

53)

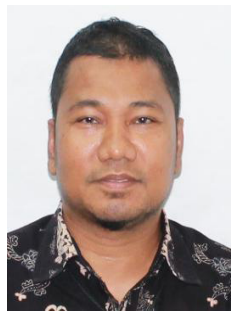

36)

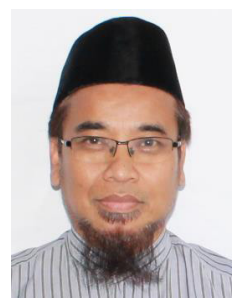

42)

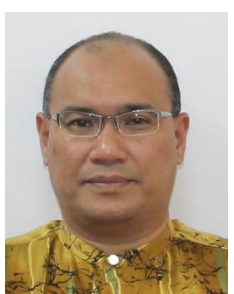

48)

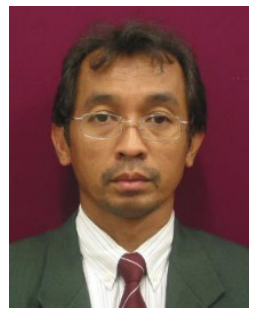

54)

Figure 7. Members of Brain and Behaviour Cluster, [previously known as P3Neuro (Pusat Perkhidmatan dan Penyelidikan Neurosains)], School of Medical Sciences, Universiti Sains Malaysia 
1) Professor Dato' Dr Jafri Malin AbdullahChairman

2) Professor Dr Zamzuri Idris-Deputy Chairman

3) Associate Professor Madya Dr Asrenee Ab. Razak-Deputy Chairman

4) Associate Professor Dr Azizah OthmanDeputy Chairman

5) Associate Professor Dato' Dr Ab Rahman Izaini Ghani @ Ab. Ghani

6) Associate Professor Dr Abdul Aziz Mohamed Yusoff

7) Associate Professor Dr Muzaimi Mustapha

8) Dr Regunath a/l Kandasamy

9) Dr Mohammed Faruque Reza

10) Dr Tahamina Begum

11) Dr Farizan Ahmad

12) Dr Muhammad Hafiz Hanafi

13) Dr Mohd Nasir Che Mohd Yusoff

14) Dr Mohamed Faiz Mohamed Mustafar

15) Dr Aini Ismafairus Abd Hamid

16) Dr Mohd Zulkifli Mustafa

17) Dr Sangu Muthuraju

18) Dr Mohd Nor Azim Ab Patar

19) Dr Sabarisah Hashim

20) Ms Nurul Aini M Nashir

21) Ms Nur Amalina Hashim

22) Mr Hazim Omar

23) Mdm Mazira Mohamad Ghazali

24) Mdm Alwani Liyana Ahmad

25) Mdm Wan Nor Azlen Wan Mohamad

26) Ms Kee Sui Mei

27) Mdm Nuraza Othman
28) Ms Nur Azza Husna Ahmad Satar

29) Mdm Nur Faten Hamzah

30) Mdm Sharifah Aida Shekh Ibrahim (Research Officer))

31) Mdm Athirah Raihanah Abdul Wahab (Science Officer)

32) Mr Mohd Eilham Yusof

33) Dr Sanihah Abdul Halim

34) Associate Professor Dr Salmi Ab Razak

35) Associate Professor Dr Zahiruddin Othman

36) Dr Mohd Azhar Mohd Yasin

37) Dr Maruzairi Husain

38) Dr Norzila Zakaria

39) Dr Sharifah Zubaidiah Syed Jaapar

40) Dr Nor Asyikin Fadzil

41) Dr Raishan Shafini Bakar

42) Associate Professor Dr Mohd Shafie Abdullah

43) Dr Chandran a/l Nadarajan

44) Dr Nik Fariza Husna Nik Hassan

45) Cik Aimi Syahidah Zulkipli

46) Dr W Mohd Nazaruddin W Hassan

47) Dr Mohamad Hasyizan Hassan

48) Professor Dr Wan Hazabbah Wan Hitam

49) Professor Dr Rosni Abdullah @ Mustafa

50) Associate Professor Dr Putra Sumari

51) Dr Nurul Hashimah Ahamed Hassain Malim

52) Dr Nur Intan Raihana Ruhaiyem

53) Dr Nur Syibrah Muhamad Naim

54) Professor Dr Mohd Zaid Abdullah

55) Associate Professor Dr Haidi Ibrahim

56) Dr Anusha a/p Achuthan 
Editorial | Neurosciences Malaysia global precision brain health
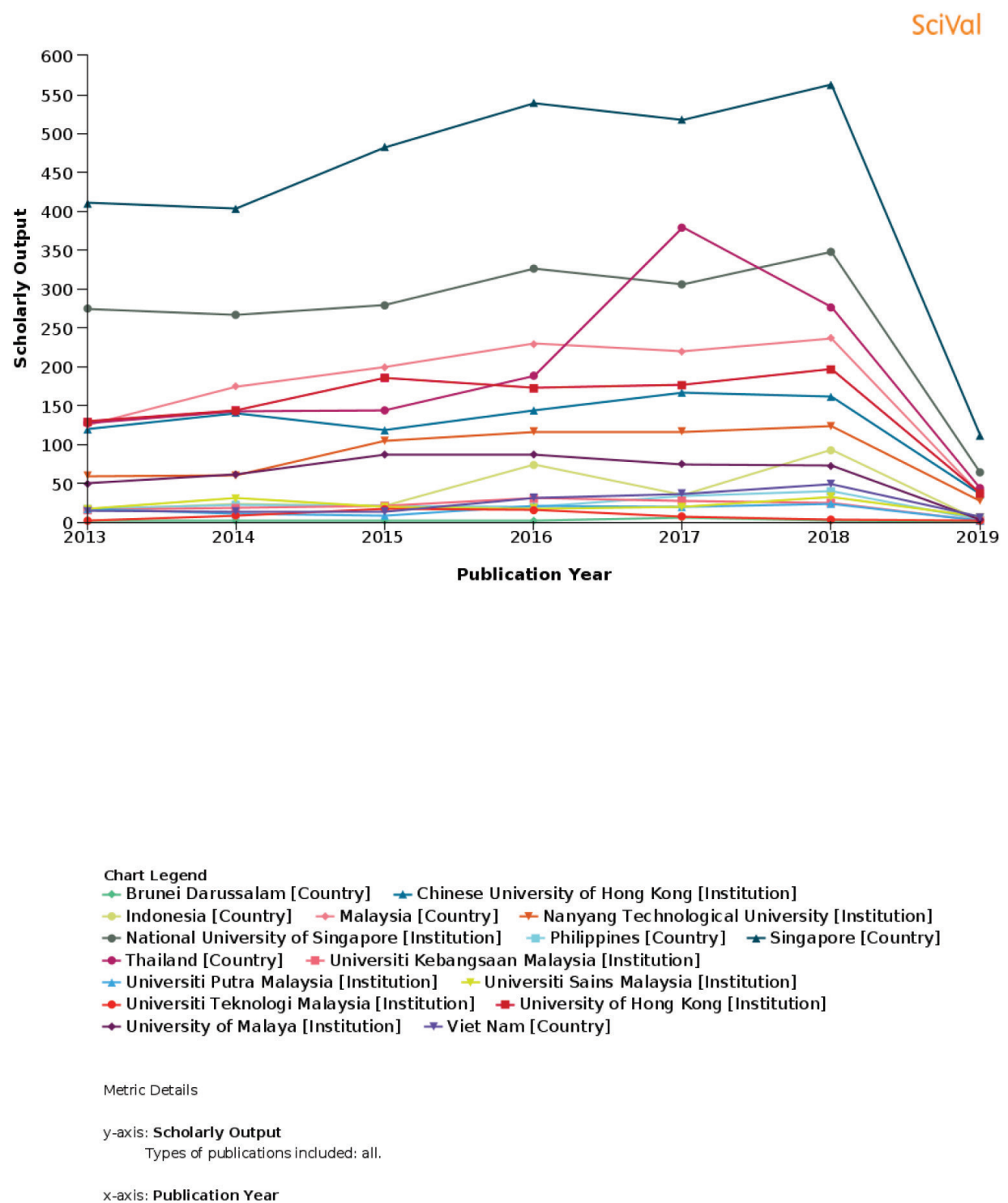

Figure 8. USM ranking in neuroscience according to SciVal, Elsevier

First ranking: Universiti Malaya

Second ranking: Universiti Sains Malaysia

Third ranking: Universiti Kebangsaan Malaysia

Fourth ranking: Universiti Putra Malaysia

Fifth ranking: Universiti Teknologi Malaysia 

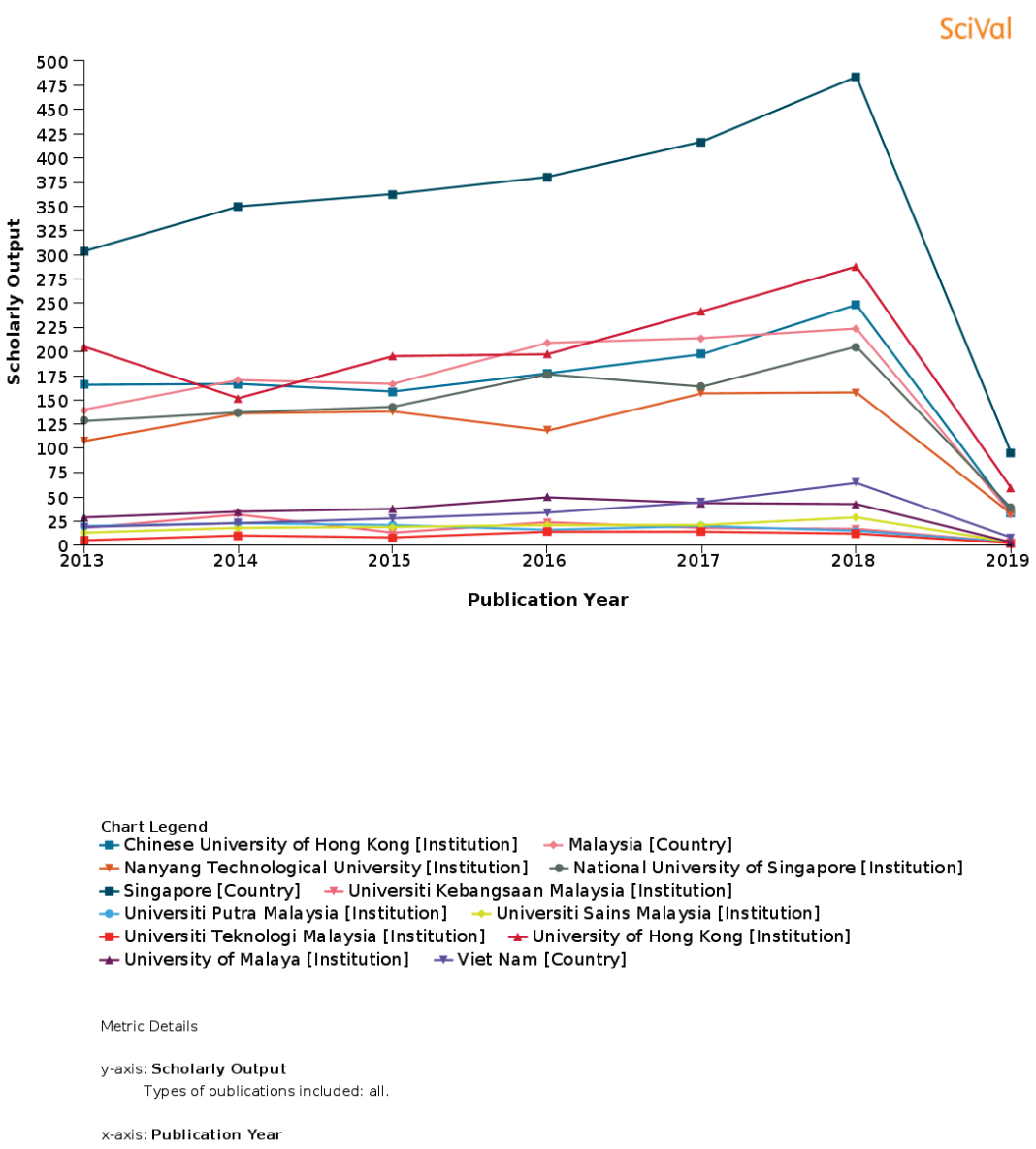

Figure 9. USM ranking in psychology according to SciVal, Elsevier

First ranking: Universiti Malaya

Second ranking: Universiti Sains Malaysia

Third ranking: Universiti Kebangsaan Malaysia

Fourth ranking: Universiti Putra Malaysia

Fifth ranking: Universiti Teknologi Malaysia

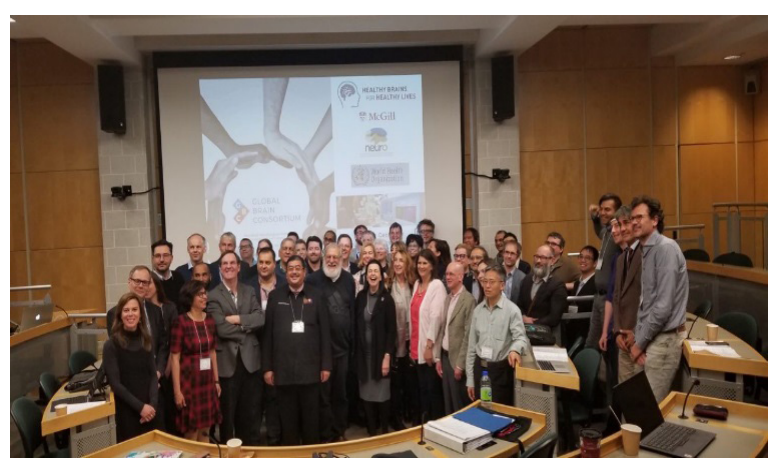

(a)

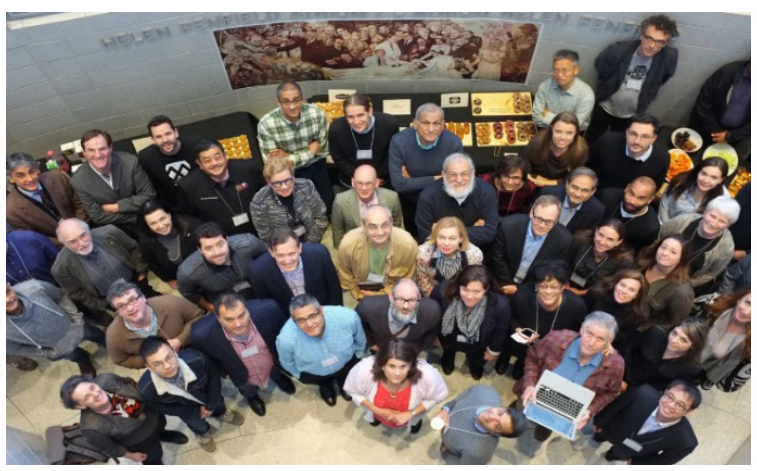

(b)

Figure 10. Pictures show Professor Alan Evan (a) front row, stood third from left, (b) at the back, stood second from left] and Professor Pedro Valdes Sosa (a) front row, stood fifth from left, (b) forth row, stood fifth from right], and the founders involved in the consortium 


\section{What We Strive For}

Mental and neurological disorders account for an increasing proportion of the global burden of disease. Additionally, the accessibility and affordability of appropriate treatment remains low, especially in countries classified as LMIC by the World Bank, where approximately $85 \%$ of the world's population resides. To improve mental health services, LMICs must increase their workforces, particularly the number of trained professionals who can provide mental health services. Although primary health care professionals can provide the bulk of care, mental health professionals-namely psychiatrists, nurses and psychosocial health experts-are needed to manage patients who are referred for specialised care and to deliver training, support and supervision to non-specialists. Without these mental health professionals, LMICs will be unable to meet their populations' mental health treatment requirements. Advanced technicians would also be involved in the development of EEG policies, procedures, provision of EEG-related supervision, and training of less-experienced EEG technicians and staff (2-6).

\section{Conclusion}

The neuroscience group, in collaboration with psychiatry and psychology, hopes to further the global WHO agenda and envisions its implementation in the next three years. We hope for support from the Academy of Sciences Malaysia, universities, the Malaysian Ministry of Health and the public to make this endeavour a success.

The teacher who is indeed wise does not bid you to enter the house of his wisdom but rather leads you to the threshold of your mind-Khalil Gibran.

\section{Correspondence}

Professor Dato' Dr Jafri Malin Abdullah MD, PhD, FRCS (Ed), FACS, DSCN (Belgium)

Chief Editor

The Malaysian Journal of Medical Sciences, Universiti Sains Malaysia, 16150 Kubang Kerian Kelantan, Malaysia.

Tel: +6097676300

Fax: +609 7673833

E-mail: brainsciences@gmail.com

\section{References}

1. Koplan J, Bond TC, Merson MH, Reddy KS, Rodriguez MH, Sewankambo NK, et al. Towards a common definition of global health. Lancet. 2009;373(9679):1993-1995. https://doi.org/10. 1016/So140-6736(09)60332-9

2. Abdullah JM. Neuroscience in Universiti Sains Malaysia: the way to go forward in Malaysia with vision 2020. Malays J Med Sci. 2005;12(2):1-3.

3. Abdullah JM. Another important news from the neuronman: Malaysia's Neuroscience Group moves upwards in terms of research, creativity and innovation. Malays J Med Sci. 2015;22(Spec Issue):1-4.

4. Putra S, Zamzuri I, Jafri Malin A. We must invest in applied knowledge of computational neuroscience and neuroinformatics as important future in Malaysia: the Malaysian Brain Mapping Project. Malays J Med Sci. 2017;24(1):1-9. https://doi.org/10.21315/mjms2017.24.1.1

5. Abdullah JM. Hominem sine opus spatium: where do the ideas come from to move the brain, mind, behaviour and neurosciences in Malaysia? Malays J Med Sci. 2018;25(2):1-14. https://doi. org/10.21315/mjms2018.25.2.1

6. Academy of Sciences Malaysia. Science and technology foresight Malaysia 2050: emerging science, engineering and technology (ESET) study. Kuala Lumpur: Academy of Science Malaysia; 2017. 